\title{
Oxysterols Present in Alzheimer's Disease Braininduce Synaptoxicity by Activating Astrocytes: A Major Role for Lipocalin-2
}

Erica Staurenghi ( $\nabla$ erica.staurenghi@unito.it)

Department of Clinical and Biological Sciences, University of Turin, Turin, Italy https://orcid.org/00000001-5035-7401

\section{Valentina Cerrato}

Department of Clinical and Biological Sciences, University of Turin, Turin, Italy

\section{Paola Gamba}

Department of Clinical and Biological Sciences, University of Turin, Turin, Italy

\section{Gabriella Testa}

Department of Clinical and Biological Sciences, University of Turin, Turin, Italy

\section{Serena Giannelli}

Department of Clinical and Biological Sciences, University of Turin, Turin, Italy

\section{Valerio Leoni}

Department of Medicine and Surgery, University of Milan-Bicocca, Desio, Monza-Brianza (MB), Ital

\section{Claudio Caccia}

Fondazione IRCCS Istituto Neurologico Carlo Besta

\section{Annalisa Buffo}

Unit of Medical Genetics and Neurogenetics, Fondazione IRCCS IstitutoNeurologico Carlo Besta, Milan, Italy

\section{Wendy Noble}

Institute of Psychiatry, Psychology and Neuroscience, Department of Basic and Clinical Neuroscience, King's College London, London, UK

\section{Beatriz Gomez Perez-Nievas}

Institute of Psychiatry, Psychology and Neuroscience, Department of Basic and Clinical Neuroscience, King's College London, London, UK

\section{Gabriella Leonarduzzi}

Department of Clinical and Biological Sciences, University of Turin, Turin, Ital

\section{Research article}

Keywords: Oxysterols, Astrocytes, Astrocyte reactivity, Lipocalin-2, Neurons, Synapses, Synaptotoxicity, Alzheimer's disease 
Posted Date: August 14th, 2020

DOI: https://doi.org/10.21203/rs.3.rs-52307/v1

License: (c) (i) This work is licensed under a Creative Commons Attribution 4.0 International License. Read Full License 
1 Oxysterols present in Alzheimer's disease brain induce synaptoxicity by activating astrocytes: a major role for lipocalin-2

4 Erica Staurenghi ${ }^{1 *}$, Valentina Cerrato ${ }^{2,3}$, Paola Gamba ${ }^{1}$, Gabriella Testa $^{1}$, Serena Giannelli ${ }^{1}$, Valerio

5 Leoni $^{4}$, Claudio Caccia ${ }^{5}$, Annalisa Buffo ${ }^{2,3}$, Wendy Noble ${ }^{6 \S}$, Beatriz Gomez Perez-Nievas ${ }^{6 \S}$, 6 Gabriella Leonarduzzi ${ }^{1 \S}$.

${ }^{1}$ Department of Clinical and Biological Sciences, University of Turin, Turin, Italy.

9 erica.staurenghi@unito.it, paola.gamba@unito.it, gabriella.testa@unito.it, serena.giannelli@unito.it,

10 gabriella.leonarduzzi@unito.it

$11{ }^{2}$ Department of Neuroscience Rita Levi-Montalcini, University of Turin, Turin, Italy. 12 valentina.cerrato@unito.it, annalisa.buffo@unito.it

$13{ }^{3}$ Neuroscience Institute Cavalieri Ottolenghi, Orbassano, Turin, Italy. valentina.cerrato@unito.it, 14 annalisa.buffo@unito.it

$15{ }^{4}$ Department of Medicine and Surgery, University of Milan-Bicocca, Desio, Monza-Brianza (MB),

16 Italy.valerio.leoni@unimib.it

$17{ }^{5}$ Unit of Medical Genetics and Neurogenetics, Fondazione IRCCS Istituto Neurologico Carlo Besta, 18 Milan, Italy. claudio.caccia@istituto-besta.it

$19{ }^{6}$ Institute of Psychiatry, Psychology and Neuroscience, Department of Basic and Clinical 20 Neuroscience, King's College London, London, UK. wendy.noble@kcl.ac.uk, beatriz.gomez_perez-

21 nievas@kcl.ac.uk

22 *Corresponding author: Erica Staurenghi erica.staurenghi@unito.it 
$23 \S$ Wendy Noble, Beatriz Gomez Perez-Nievas, and Gabriella Leonarduzzi equally contributed to this 24 work and are joint last authors.

\section{Abstract}

Background: Among Alzheimer's disease (AD) brain hallmarks, the presence of reactive astrocytes was demonstrated to correlate with neuronal loss and cognitive deficits. Evidence indeed supports the role of reactive astrocytes as mediators of changes in neurons, including synapses. However, the complexity and the outcomes of astrocyte reactivity are far from being completely elucidated.

31 Another key role in AD pathogenesis is played by alterations in brain cholesterol metabolism. Oxysterols (cholesterol oxidation products) are crucial for brain cholesterol homeostasis, and we previously demonstrated that changes in the brain levels of various oxysterols correlate with AD progression. Moreover, oxysterols have been shown to contribute to various pathological mechanisms involved in $\mathrm{AD}$ pathogenesis.

In order to deepen the role of oxysterols in $\mathrm{AD}$, we investigated whether they could contribute to astrocyte reactivity, and consequently impact on neuronal health.

Methods: Mouse primary astrocyte cultures were used to test the effect of two oxysterol mixtures, that represent the oxysterol composition respectively of mild or severe AD brains, on astrocyte morphology, markers of reactivity, and secretion profile. Co-culture experiments were performed to

41 investigate the impact of oxysterol-treated astrocytes on neurons. Neuronal cultures were exposed to astrocyte conditioned media (ACM) deprived of lipocalin-2 ( $\mathrm{ccn} 2)$ to investigate the contribution of this mediator to synaptotoxicity.

Results: Results showed that oxysterols induce a clear morphological change in astrocytes, accompanied by the upregulation of some reactive astrocyte markers, including Lcn2. Moreover, ACM analysis revealed a significant increase in the release of Lcn2, cytokines, and chemokines in 
response to oxysterols. A significant reduction of postsynaptic density protein 95 (PSD95) and a concurrent increase in cleaved caspase-3 protein levels have been demonstrated in neurons cocultured with oxysterol-treated astrocytes, pointing out that mediators released by astrocytes have an impact on neurons. Among these mediators, Lcn2 has been demonstrated to play a major role on synapses, affecting neurite morphology and decreasing dendritic spine density.

Conclusions: These data demonstrated that oxysterols present in the AD brain promote astrocyte reactivity, determining the release of several mediators that affect neuronal health and synapses. Lcn2 has been shown to exert a key role in mediating the synaptotoxic effect of oxysterol-treated astrocytes.

Keywords: Oxysterols; Astrocytes; Astrocyte reactivity; Lipocalin-2; Neurons; Synapses; Synaptotoxicity; Alzheimer's disease.

\section{BACKGROUND}

Alzheimer's disease $(\mathrm{AD})$ is a neurodegenerative disorder, that represents the most common form of dementia and affects millions of people worldwide [1]. The pathogenic hallmarks of AD are extracellular deposits of amyloid- $\beta$ (A $\beta$ ) peptides in the form of senile plaques and intracellular neurofibrillary tangles (NFTs) made of hyperphosphorylated tau protein. These lesions are typically accompanied by gliosis, especially surrounding senile plaques [2]. Neuronal death and synapse loss are also observed; in particular, synaptic loss exceeds neuronal loss, meaning that remaining neurons also lose synapses. Synapse loss is indeed the best correlate of cognitive decline [3].

Astrocytes play a crucial role in maintaining brain homeostasis and their reaction to different kind of insults leads to a heterogeneous range of changes, known as "astrocyte reactivity" [4]. An increase in the number of reactive astrocytes is a typical histopathological feature of AD brain, and it correlates with cognitive decline and neuronal loss also in transgenic mouse models $[5,6]$. Besides 
morphological changes and the upregulation of common markers of reactivity (e.g. the glial fibrillary acidic protein, GFAP), reactive astrocytes show significant alterations in gene expression and functions, depending on the specific stimulus $[7,8]$. However, the outcome of astrocyte reactivity is still somewhat controversial. Several studies highlighted that reactive astrocytes lose neuroprotective functions, including their ability to promote neurite growth, neuronal survival, and synapse formation $[9,10]$. One of the mechanisms through which astrocytes could have an impact on neurons is by the altered release of different kinds of molecules including cytokines, chemokines, growth factors, and neurotransmitters. For instance, an increase in the release of inflammatory mediators by reactive astrocytes has been shown to affect neuronal viability, tau phosphorylation $[9,11]$, and synaptic function $[12,13]$. Another mediator released by reactive astrocytes is lipocalin-2 (Lcn2), a protein identified as a pan-reactive astrocyte marker [8]. Lcn2 is a member of the lipocalin protein family, consisting of more than 20 proteins that transport small hydrophobic molecules (e.g steroids, lipids, and retinoids) and it plays important roles in the immune response, cell migration and proliferation [14]. Increased levels of Lcn2 have been found in the entorhinal cortex and hippocampus of AD brain [15]. Emerging evidence indicates that Len2 in the brain is synthethized and secreted as an inducible factor by activated microglia, reactive astrocytes, neurons, and endothelial cells in response to inflammatory stimuli, infections or other insults $[16,17]$.

In addition to amyloid plaques and NFTs, Alois Alzheimer originally described the presence of "adipose inclusions" in the glial cells of $\mathrm{AD}$ brains, suggesting a malfunctioning of lipid metabolism [18]. A clear link between AD and lipid metabolism was established later by the identification of the $\varepsilon 4$ allele of apolipoprotein $\mathrm{E}(\mathrm{ApoE})$ as a strong genetic risk factor for $\mathrm{AD}$ [19]. Moreover, in the last few decades, much other evidence supports a role for lipids, in particular cholesterol, in $\mathrm{AD}$ pathogenesis [20-22]. As the main lipid component of neuronal and glial membranes, as well as a key constituent of myelin, cholesterol plays an essential role in synapse formation, maintenance, and function [23, 24]. Astrocytes are the main producers of cholesterol in 
the brain, that is delivered to neurons loaded into ApoE-containing lipoproteins. Brain cholesterol homeostasis is closely controlled by pathways regulating cholesterol biosynthesis, storage, and elimination; in particular, the main process responsible for cholesterol elimination is enzymatic oxidation to oxysterols that are able to cross the blood-brain barrier (BBB). Excess brain cholesterol is essentially oxidized to 24-hydroxycholesterol (24-OHC) by cholesterol 24-hydroxylase (CYP46A1), a member of the cytochrome P450 family mainly expressed by neurons; the additional hydroxyl group enables 24-OHC to flow into the circulation crossing the BBB [25]. Smaller amounts of other enzymatic oxysterols, such as 27-hydroxycholesterol (27-OHC), can be produced in the brain, as well as various oxysterols deriving from cholesterol non-enzymatic oxidation mediated by various compounds (e.g. free radical species, metal cations, and $A \beta$ peptides) $[26,27]$. Oxysterols are not only cholesterol metabolites but they also play many regulatory functions, such as modulating cholesterol biosynthesis, inflammatory pathways, and the immune response [28]. We have previously demonstrated a correlation between changes in brain levels of various oxysterols and AD progression. Through analysis of the oxysterol composition in AD and aged-matched control cortex, we observed a significant increase in the levels of various enzymatic (e.g. 27-OHC) and non-enzymatic oxysterols (e.g. 7 $\alpha$-hydroxycholesterol, 7 $\alpha$-OHC; 7 $\beta$-hydroxycholesterol, 7 $\beta$-OHC; 7-ketocholesterol, 7-KC; 5 $\alpha, 6 \alpha$-epoxycholesterol, $\alpha$-EPOX; $5 \beta, 6 \beta$-epoxycholesterol, $\beta$-EPOX). In contrast, 24-OHC levels were markedly decreased compared to control brains, probably due to the decline in CYP46A1 expression levels as a result of neuronal loss [27]. The effects of oxysterols are still controversial but growing evidence suggests that some of them (e.g. $27-\mathrm{OHC}, 7-\mathrm{KC}, 7 \alpha-$ and $7 \beta-\mathrm{OHC}$ ) may play a role in $\mathrm{AD}$ pathogenesis by inducing oxidative stress, inflammation [29], $\mathrm{A} \beta$ formation and accumulation [30, 31], tau hyperphosphorylation [32], synaptic dysfunction [33], and cell death [34, 35].

At present, data regarding the impact of oxysterols on astrocytes are limited and mostly related to the ability of some of them to affect brain cholesterol synthesis and transport in various astrocytic 
121 cell lines [36, 37]. For instance, it has been shown that an increase in systemic levels of 27-OHC can

122 disrupt brain cholesterol homeostasis in rats by reducing cholesterol synthesis and increasing its

123 efflux, but also favouring brain cholesterol accumulation likely due to cell damage; moreover, spatial

124 learning and memory deficits were described as a result [38]. 27-OHC was also observed to induce 125 oxidative stress and to downregulate the antioxidant response in C6 glioma cells, leading to cell 126 toxicity [39]. 24-OHC has been shown to affect redox homeostasis in human glial cells, although its 127 impact may depend on the concentration [40]. Interestingly, the oxysterols $7-\mathrm{KC}, 7 \alpha-\mathrm{OHC}$, and $7 \beta-$ 128 OHC have been shown to inhibit cell growth and decrease viability in several neuronal and glial cell 129 lines; in particular, 7-KC and 7 $\beta-\mathrm{OHC}$ exhibited cytotoxic effects also in mixed glial murine primary 130 cultures [35]. High concentrations of $7 \beta-\mathrm{OHC}$ have also been observed to induce toxic effects and 131 morphological changes in an in vitro model of reactive astrocytes [41].

Since growing data support the involvement of oxysterols in several aspects of AD pathology 133 and given the presence of reactive astrocytes in the disease, we wanted to investigate whether these 134 compounds could impact on astrocyte reactivity, potentially compromising neuronal health. With the 135 aim of mimicking the human $\mathrm{AD}$ brain oxysterol composition, we used two oxysterol mixtures, both 136 including the main seven oxysterols previously quantified in cortical AD brain samples and 137 representative of early or late stages of the disease [27], to investigate the effect of oxysterols in 138 mouse cortical astrocytes and neuronal cultures.

\section{METHODS}

\section{Composition of oxysterol mixtures}

142 Cell cultures were treated with two oxysterol mixtures, whose compositions represent oxysterol 143 amounts previously quantified in mild (Early AD mixture) or severe (Late AD mixture) AD brain 144 samples [27]. Both oxysterol mixtures consist of the same seven oxysterols but in different 
proportions. Early AD mixture composition: 24-OHC (52.9\%), 27-OHC (3\%), 7-KC (9.2\%), 7 $\alpha-$ $\mathrm{OHC}(4.5 \%), 7 \beta-\mathrm{OHC}(19.2 \%), \alpha$-EPOX (3\%), and $\beta$-EPOX (8.2\%). Late AD mixture composition: 24-OHC (33.4\%), 27-OHC (5.8\%), 7-KC (12.7\%), 7 $\alpha$-OHC (5.4\%), 7 $\beta$-OHC (23.8\%), $\alpha$-EPOX (4.9\%), and $\beta$-EPOX (14\%). Oxysterols were dissolved in absolute ethanol. Lipids, Alabaster, AL, USA), $\alpha$-EPOX (C4130-000), and $\beta$-EPOX (C5030-000) (Steraloids, Newport, RI, USA).

\section{Primary cultures and co-cultures}

Primary astrocyte cultures were obtained from cerebral cortex of wild type CD1 mice on postnatal day 1-3 as previously described [42]. Briefly, after dissociation of the cortices, cells were resuspended in growing medium (DMEM high glucose with glutaMAX, sodium pyruvate, $10 \%$ fetal bovine serum, $100 \mathrm{U} / \mathrm{ml}$ penicillin, $100 \mu \mathrm{g} / \mathrm{ml}$ streptomycin) and seeded into T75 flasks, previously coated with poly-D-lysine $(10 \mu \mathrm{g} / \mathrm{ml})$ for at least $1 \mathrm{~h}$ at $37^{\circ} \mathrm{C}$. Astrocytes were cultured for $7-14$ days in a humidified $5 \% \mathrm{CO}_{2}$ incubator at $37^{\circ} \mathrm{C}$, shaking them at $200 \mathrm{rpm}$ overnight on days 3 and 7 to remove microglia and oligodendrocytes. The absence of microglial contamination was assessed by immunocytochemistry and Western blotting using antibodies that recognize the ionized calcium binding adaptor molecule 1 (Iba1, microglial marker) (Supplemental Fig. 1). Astrocyte-enriched cultures were then re-plated into 12-wells plates using trypsin and the medium was changed to Neurobasal serum-free medium (supplemented with 2\% B-27, 2 mM glutaMAX, 100 U/ml penicillin,

$166100 \mu \mathrm{g} / \mathrm{ml}$ streptomycin) $24 \mathrm{~h}$ before treatment. Astrocyte cultures were treated with the oxysterol mixtures (Early or Late at different concentrations) up to 24h. Vehicle (ethanol) was added to control astrocytes. 
Primary neurons were obtained from cerebral cortex of wild type CD1 mouse embryos at embryonic day 15, according to a previously published protocol [43]. Briefly, after dissociation, cortices were washed twice in HBSS (without $\mathrm{Ca}^{2+}$ and $\mathrm{Mg}^{2+}$ ) and homogenized in $1 \mathrm{ml}$ of Neurobasal serum-free medium (supplemented with 2\% B-27, $2 \mathrm{mM}$ glutaMAX, $100 \mathrm{U} / \mathrm{ml}$ penicillin, $100 \mu \mathrm{g} / \mathrm{ml}$ streptomycin). The suspension was then filtered using a $40 \mu \mathrm{m}$ cell strainer, live cells were counted and neurons were plated to a density $5 \times 10^{5}$ viable cells per well of 6-wells plates previously coated with poly-D-lysine $(10 \mu \mathrm{g} / \mathrm{ml})$ for at least $1 \mathrm{~h}$ at $37^{\circ} \mathrm{C}$. After $1 \mathrm{~h}$, neuron adhesion was checked and the medium was changed. Cultures were maintained at $37^{\circ} \mathrm{C}$ with $5 \% \mathrm{CO}_{2}$ in Neurobasal serum-free medium. Neuronal cultures were treated at 13-14 days in vitro (DIV). For neurite and spine analysis, neurons were transfected at 5-7 DIV with the plasmid peGFP-N1 (Clontech, Mountain View, CA, USA) using Lipofectamine 2000 (Invitrogen, Thermo Fisher Scientific, Waltham, MA, USA) and imaged live $24 \mathrm{~h}$ after treatment at 14 DIV using an Opera Phenix microscope (Perkin Elmer, Waltham, MA, USA).

For co-culture experiments, primary astrocytes were plated into cell-culture inserts $(0.4 \mu \mathrm{m}$ pore membrane, Falcon, Corning, Corning, NY, USA), that allow the passage of small molecules in culture medium. Astrocytes were pre-treated with the Late AD mixture $(10 \mu \mathrm{M})$ or vehicle (ethanol) for $12 \mathrm{~h}$, medium was changed to remove oxysterols, and inserts on which astrocytes were grown were added to neuron cultures for a further $24 \mathrm{~h}$.

\section{Cytotoxicity assay}

189 Cytotoxicity was assessed by measuring levels of lactate dehydrogenase (LDH) enzyme release into 190 the media using Pierce LDH Cytotoxicity Assay Kit (Fisher Scientific, Thermo Fisher Scientific) 191 according to the manufacturers' instructions. Some cells were lysed with $0.5 \%$ Triton X-100 and LDH content in the medium was measured in order to evaluate the maximum LDH amount released from dead cells. LDH release was calculated as a percentage of total LDH released by lysed cells. 


\section{Gel electrophoresis and Western blotting}

After treatments, the cell culture media were collected, cells were washed with PBS and directly lysed with PBS containing sample buffer (NuPAGE LDS Sample Buffer 4X, Invitrogen), reducing agent (NuPAGE Sample Reducing Agent 10X, Invitrogen), protease inhibitor (complete Mini EDTA-Free Protease Inhibitor Cocktail, Roche, Basel, $\mathrm{CH}$ ) and phosphatase inhibitor (PhosSTOP, Roche) cocktails.

Equal amounts of protein samples $(20 \mu \mathrm{g})$ were boiled, separated by electrophoresis using $10 \%$ precast gels (NuPAGE 10\% Bis-Tris Protein Gels, Invitrogen) and then transferred to nitrocellulose membranes (Amersham Protran, GE Healthcare, Chicago, IL, USA). After blocking with Odyssey Blocking Buffer (LI-COR Biosciences, Lincoln, NE, USA) for 1h at room temperature, membranes were incubated with primary antibodies overnight at $4^{\circ} \mathrm{C}$. The following primary antibodies were used: GFAP (Z0334, Dako, Agilent Technologies, Santa Clara, CA, USA), Lipocalin-2/NGAL (AF1857, R\&D Systems, Minneapolis, MN, USA), SerpinA3N (AF4709, R\&D Systems), cleaved caspase-3 (Asp175, 9661, Cell Signaling, Danvers, MA, USA), PSD95 (D74D3, Cell Signaling), Synapsin (6008-30, BioVision, Milpitas, CA, USA), Iba1 (019-19741, Wako Chemicals, Richmond, VA, USA) and $\beta$-actin (ab8226, Abcam, Cambridge, UK). After washing to remove unbound antibody with TBS-Tween20 0.05\%, the appropriate fluorophore-coupled secondary antibody (1:10000, LI-COR Biosciences) was added for $1 \mathrm{~h}$ at room temperature. Membranes were washed with TBS-Tween20 0.05\% and scanned using an Odyssey infrared imaging system (LI-COR Biosciences). Band intensities were quantified using the Image Studio Software (LICOR Biosciences) and normalized to the corresponding $\beta$-actin bands.

Similarly, to detect secreted Lcn2, $250 \mu$ of culture media were concentrated using centrifugal filters (Amicon Ultra, Millipore, Merck, Darmstadt, DE); the protein concentration was calculated 
using the Bradford Assay (Protein Assay Dye Reagent Concentrate, Bio-Rad Laboratories, Hercules,

219 CA, USA), and samples were prepared as above to perform Western blotting of cell culture media.

\section{Immunocytochemistry}

Astrocytes were plated on coverslips (12 or $18 \mathrm{~mm}$ diameter, No. 1.5) into 12- or 24-wells plates. After treatment, cells were washed with PBS, fixed in 4\% paraformaldehyde in PBS for 10 min at room temperature, and then washed again twice with PBS. Cells were permeabilized and blocked (4\% goat serum, $0.1 \%$ Triton in PBS) for $1 \mathrm{~h}$ at room temperature before incubating with the antiGFAP (Z0334, Dako) and/or Iba1 (ab48004, Abcam) primary antibodies (4\% goat serum in PBS) overnight at $4^{\circ} \mathrm{C}$. The following day, cells were incubated with the appropriate secondary antibody conjugated with fluorescent probes for $1 \mathrm{~h}$ at room temperature (Alexa Fluor 488 or 594, Invitrogen) and nuclei were stained with Hoescht $33258(10 \mu \mathrm{g} / \mathrm{ml}$ in PBS, Sigma-Aldrich, St. Louis, MO, USA). Cells were imaged using an Eclipse Ti-E inverted Microscope (Nikon, Tokyo, JP) or LSM800 confocal microscope (Carl Zeiss, Oberkochen, DE).

\section{Cytokine array}

234 Astrocyte culture media was collected and stored at $-20^{\circ} \mathrm{C}$ prior to analysis of cytokine and chemokine content using Mouse Proteome Profiler arrays (Mouse Cytokine Array Panel A, R\&D Systems), according to the manufacturers' instructions. Briefly, array membranes were incubated in blocking

237 buffer for $1 \mathrm{~h}$ at room temperature. Each sample of medium was incubated with the Detection 238 Antibody Cocktail and this mix was then placed onto blocked membranes overnight at $4^{\circ} \mathrm{C}$. After washes, membranes were incubated with IRDye 800CW Streptavidin (1:2000, LI-COR Biosciences) for $30 \mathrm{~min}$ at room temperature. Membranes were then scanned using an Odyssey infrared imaging system and the spot intensities were quantified using the Image Studio Software (LI-COR 
Biosciences). Positive and negative control spots included in each membrane allowed quantitative analysis by densitometry and results were expressed as percentage change compared to control 244 cultures.

\section{Oxysterol quantification in astrocyte culture medium}

Astrocyte culture media $(100 \mu \mathrm{l})$ was added to a screw-capped vial sealed with a Teflon septum together with D7-7 $\alpha$-hydroxycholesterol (50 ng), D7-7 $\beta$-hydroxycholesterol (50 ng), D7-7-oxocholesterol (50 ng), D6-5 $\alpha$-6 $\alpha$-epoxicholesterol (50 ng), D6-5 $\beta$-6 $\beta$-epoxicholesterol, D6-24hydroxycholesterol (250 ng), D6-27-hydroxycholesterol (50 ng) as internal standards, as well as 50 $\mu \mathrm{l}$ of butylated hydroxytoluene $(5 \mathrm{~g} / \mathrm{l})$ and $50 \mu \mathrm{l}$ of K3-EDTA $(10 \mathrm{~g} / \mathrm{l})$ to prevent auto-oxidation. Each vial was then flushed with argon for 5 min to remove air. Alkaline hydrolysis, sterol extraction, and gas chromatography-mass spectrometry (GC-MS) analysis were performed as previously described [44].

\section{RNA extraction and real-time RT-PCR}

Total RNA was extracted using TriFast reagent (Eurogold TriFast, EuroClone, Pero, IT) following the manufacturers' instructions. RNA was dissolved in RNase-free water with RNase inhibitors (SUPERase-In RNase inhibitor, Invitrogen). The amount and purity of the extracted RNA were assessed by using a NanoDrop ND-1000 spectrophotometer (Thermo Fisher Scientific). cDNA was synthesized by reverse transcription of $1 \mu \mathrm{g}$ of RNA by using a commercial kit and random primers (High-Capacity cDNA Reverse Transcription Kit, Applied Biosystems, Thermo Fisher Scientific) following the manufacturers' instructions. 
266 Fast Universal PCR Master Mix, and a 7500 Fast Real-Time PCR System (Applied Biosystems). The 267 PCR cycling parameters were set up as previously described [31]. The fractional cycle number $(\mathrm{Ct})$ 268 was determined for each gene considered and results were then normalized to $\beta$-actin expression. 269 Relative quantification of target gene expression was achieved with a mathematical method [45].

\section{Transient gene knockdown}

The transient knockdown of Lcn2 gene was performed by using a Small Interfering RNA (siRNA) (Silencer Select Pre-designed siRNA S69122, Ambion, Thermo Fisher Scientific) following the manufacturers' instructions. A non-targeting siRNA was used as a negative control (Silencer Select Negative Control \#2 siRNA, Ambion). Briefly, astrocytes were plated into 12- or 24-wells plates and respectively $50 \mu \mathrm{l}$ or $100 \mu \mathrm{l}$ of a mix containing siRNA and transfection agent (Lipofectamine RNAiMAX Reagent, Invitrogen) in a 1:1 ratio was added to each well, in order to reach a final siRNA concentration of $100 \mathrm{nM}$ and $3 \mu \mathrm{l} / \mathrm{ml}$ of transfection agent. After $6 \mathrm{~h}$ transfection, the medium was changed and astrocytes were treated with the Late AD mixture $(10 \mu \mathrm{M})$ for $12 \mathrm{~h}$. After treatment, cells were washed with PBS and fresh medium was added. Astrocyte conditioned media were then collected after another $24 \mathrm{~h}$. Protein and RNA extraction, as well as real-time RT-PCR, were performed as described above. The silencing efficiency, validated by real-time RT-PCR, was approximately 85\% (Supplemental Fig. 2).

\section{Analysis of neuronal morphology}

The neuronal morphology of 14 DIV neurons previously transfected with the plasmid peGFP-N1

(Clontech) on 5-7 DIV and treated on 13 DIV was assessed using high resolution digital images of analysed using Harmony software: total and maximum neurite length, and number of nodes and 
extremities were quantified and compared between groups. NeuronStudio software (CNIC, Mount

291 Sinai School of Medicine) was used for dendritic spine analysis. Spine density was defined as number 292 of spines per micrometer of dendrite length. Dendritic spine densities were calculated from 20 293 neurons/condition.

\section{Statistical analysis}

After performing a Shapiro-Wilk normality test, data were analysed using one-way ANOVA followed by Bonferroni post hoc test or Student's t-test (GraphPad Prism 7 Software, Graphpad Software, La Jolla, CA, USA). Results were considered statistically significant when $\mathrm{P}<0.05$. Data are represented as means \pm standard deviation.

RESULTS

\section{Oxysterol mixtures induce a morphological change in astrocytes}

Astrocyte reaction to certain stimuli is defined by a wide range of heterogeneous responses, including changes in morphology $[7,46]$. To analyse the effect of oxysterols on astrocyte morphology and survival, cells were exposed to increasing concentrations of the Early and Late mixtures (1, 5 or 10 $\mu \mathrm{M})$. Exposure of primary astrocytes to 5 or $10 \mu \mathrm{M}$ of both oxysterol mixtures for $24 \mathrm{~h}$ induced a clear change in their morphology with astrocytes adopting a more stellate "brain-like" and reactive appearance rather than the large, flat fibroblast-like morphology typical of unstimulated dissociated astrocytes in culture (Fig. 1B). Importantly, this was not accompanied by cell death since there were

311 no significant increases in the abundance of LDH released into culture medium following treatment 312 when compared to control cultures (Fig. 1A). Time course treatments showed that astrocytes started 313 to display the typical signs of reactivity (increase in the number of GFAP positive processes and 
stellate appearance) after $12 \mathrm{~h}$ of exposure to $10 \mu \mathrm{M}$ of both Early and Late mixtures, with these

315 changes becoming more evident $24 \mathrm{~h}$ after treatment (Fig. 2).

Oxysterol mixtures increase the synthesis of the pan-reactive astrocyte markers Lcn2 and SerpinA3N

To validate the induction of astrocytic reactivity by oxysterols, and to confirm that the morphological alteration is accompanied by functional changes, we determined whether treatment with the Early or Late $\mathrm{AD}$ mixtures led to an increase in the levels of some pan-reactive astrocyte markers [8]. Exposure to both oxysterol mixtures $(10 \mu \mathrm{M})$ resulted in a marked increase in Lcn2 and serine protease inhibitor $\mathrm{A} 3 \mathrm{~N}($ SerpinA3N) protein levels at $12 \mathrm{~h}(\mathrm{P}<0.0001$ and $\mathrm{P}<0.001)$ and $24 \mathrm{~h}$ $(\mathrm{P}<0.0001)$ (Fig. 3), contemporaneous with morphological changes. These molecules are involved in the acute-phase response and were both identified as new reactive astrocyte markers [8, 47]. Total levels of GFAP were only slightly increased $(\mathrm{P}<0.05)$ or remained unchanged in response to the mixtures (Fig. 3), suggesting that the observed morphological changes are not only dependent on the expression of GFAP.

Oxysterol-induced reactivity triggers the release of Lcn2, cytokines, and chemokines by astrocytes

Astrocyte reactivity is typically characterized by the release of cytokines, chemokines, and other mediators, that could have several effects on the surrounding cells [48]. Therefore, we investigated whether oxysterol-mediated astrocyte reactivity was accompanied by altered release of mediators into the cell culture medium.

Since Lcn2 is secreted by reactive astrocytes [17, 49], we analysed its protein levels in astrocyte conditioned media (ACM) by Western blotting. We saw that the robust increase in 
338 intracellular levels of Lcn2 is accompanied by a significant increase in the secretion of Lcn 2 after $24 \mathrm{~h}$ treatment with $10 \mu \mathrm{M}$ of both Early and Late oxysterol mixtures $(\mathrm{P}<0.0001)$ (Fig. 4A).

The amounts of 40 cytokines and chemokines in ACM samples were next evaluated using

341 Proteome Profiler antibody arrays. The analysis showed a significant increase in the release of several 342 mediators by astrocytes treated for $24 \mathrm{~h}$, especially with the Late AD oxysterol mixture $(10 \mu \mathrm{M})$. Some 343 of the cytokines found to be increased in the medium of astrocytes treated with this oxysterol mixture 344 were interleukin-1 $\beta$ (IL-1 $\beta$ ), soluble intercellular adhesion molecule-1 (sICAM-1) $(\mathrm{P}<0.001)$, IL-1 $\alpha$, 345 IL-5, granulocyte colony-stimulating factor (G-CSF), and C-C motif chemokine 12 (CCL12) 346 (P<0.01). In addition, the levels of CCL1, IL-7, IL-10, IL-13, IL-16, IL-17, and C-X-C motif 347 chemokine 9 (CXCL9) were slightly but significantly increased compared to levels in control media $348(\mathrm{P}<0.05)($ Fig. 4B, C). In contrast, when astrocytes were exposed to the Early AD oxysterol mixture $349(10 \mu \mathrm{M})$, only sICAM-1 $(\mathrm{P}<0.01)$, IL-1 $\alpha$, and IL-1 $\beta(\mathrm{P}<0.05)$ were found to be significantly increased 350 relative to levels in control ACM, highlighting a different impact of the two oxysterol mixtures on 351 astrocyte cytokine and chemokine release (Fig. 4C).

\section{Oxysterol-stimulated astrocytes compromise neuronal health}

It has previously been shown that astrocyte reactivity depends on the specific stimulus and that heterogeneous responses of reactive astrocytes involves different signaling pathways. The consequences of these alterations for astrocytic support of neuronal health requires further investigation [7, 47].

To test the effect of oxysterol-stimulated astrocytes on neuronal health, we performed coculture experiments. GC-MS analysis of oxysterols in ACM showed that, although variable, a 360 substantial proportion (8-54\%) of some oxysterols remain in ACM 24h after treatment (Supplemental 361 Fig. 3). Since these oxysterols are known to directly affect neuron viability [31, 33, 50], we co- 
cultured neurons with astrocytes that had been pre-treated with oxysterols. As shown in Figure 5A, 363 astrocytes grown on cell culture inserts were pre-treated for $12 \mathrm{~h}$ with the Late AD oxysterol mixture $364(10 \mu \mathrm{M})$ and then transferred to culture plates containing neurons for a further $24 \mathrm{~h}$ : this allowed us 365 to investigate the effect of mediators released from stimulated astrocyte on neurons, without 366 transferring oxysterols that remained in ACM. In parallel, neuronal cultures were treated with the 367 Late $\mathrm{AD}$ mixture or with ethanol for $24 \mathrm{~h}$ in the absence of astrocytes, in order to assess the direct 368 effect of the same oxysterol mixture on neurons.

Interestingly, Western blotting analysis showed a significant reduction of postsynaptic density protein 95 (PSD95) levels $(\mathrm{P}<0.05)$, a scaffold protein important for postsynaptic density structure 371 and function, in neurons cultured with astrocytes that had been previously exposed to oxysterols (Fig. 5B). This reduction was accompanied by a significant increase in the amount of cleaved (active) caspase-3 $(\mathrm{P}<0.01)$ (Fig. 5B), that was demonstrated to play various non-apoptotic roles in neurons including to affect synaptic functions [51]. A mild but not significant reduction of the pre-synaptic marker synapsin was also observed. Moreover, when neurons were directly treated with oxysterols, PSD95 $(\mathrm{P}<0.05)$, cleaved caspase-3 $(\mathrm{P}<0.01)$, and synapsin levels $(\mathrm{P}<0.05)$ were all significantly altered (Fig. 5B), confirming previous results showing that oxysterols directly compromise neuron health [31, 33]. Importantly, all these results were obtained in the absence of significant neuronal death, as assessed by LDH release (Supplemental Fig. 4). Overall, these data suggest that factors oxysterol content, compromise synaptic and neuronal health without causing overt neurotoxicity. 
Lcn2 has previously been shown to affect synapses, neuronal health and morphology, [17, 52, 53].

386 To investigate whether Lcn2 could play a role in mediating the effect of oxysterol-stimulated 387 astrocytes on neurons, Lcn2 expression was silenced in astrocytes prior to determining the impact of 388 ACM on neuron morphology. To further investigate the effects of ACM on synapses under these 389 conditions, dendritic spine density was also evaluated.

For this purpose, astrocytes were transfected for $6 \mathrm{~h}$ with Lcn2 or scrambled siRNA, and the medium removed prior to treatment with the Late $\mathrm{AD}$ mixture $(10 \mu \mathrm{M})$ for $12 \mathrm{~h}$. The medium was removed and fresh medium was added for another 24h, to obtain ACM without oxysterols. Both realtime RT-PCR (Fig. 6A) and Western blotting results (Fig. 6B) confirmed that siRNA-mediated Lcn2 gene silencing prevents the increase in Lcn2 expression, synthesis, and release into the medium induced by the Late AD oxysterol mixture, compared to control conditions (scrambled siRNA). Interestingly, oxysterol-induced morphological changes were not prevented by Lcn2 gene silencing, suggesting that the pathway by which oxysterols induce astrocyte alterations is not dependent upon the presence of Lcn2 (Fig. 6C).

Next, ACM was added to cultured neurons to determine the impact of secreted Lcn2 on neuron morphology and dendritic spines. Medium from oxysterol-activated astrocytes transfected with the scrambled siRNA compromises neurite complexity, as shown by reduction of total and maximum neurite length, and the number of nodes, automatically identified by Harmony software $(\mathrm{P}<0.05)($ Fig. 403 7A). Importantly, the addition of ACM was also found to substantially reduce dendritic spine density $404(\mathrm{P}<0.001)$ (Fig. 7B), further indicating a synaptotoxic effect of proteins secreted by oxysterol405 stimulated astrocytes. All of these effects were prevented by silencing Lcn2, confirming a key role of 406 Lcn2 in mediating the impact of oxysterol-stimulated astrocytes on neurite complexity and synaptic 407 health (Fig. 7). 
410 The pathophysiology of AD is still not fully understood, but evidence supports the involvement of 411 many factors in sporadic AD onset, including inflammation and impaired cholesterol metabolism 412 [54]. Microglia and astrocytes play a role in the neuroinflammation observed in AD and they are 413 found in their activated state in affected brain regions, particularly surrounding amyloid plaques. 414 Astrocyte reactivity is not an exclusive feature of AD brain but the association of reactive astrocytes 415 with amyloid plaques is considered functionally significant. Indeed, post-mortem neuropathological 416 studies have shown that the number of reactive astrocytes increases with disease progression $[6,55]$, 417 and activated astrocytes and microglia correlate with dementia in AD [5].

Astrocyte reactivity leads to a heterogeneous range of phenotypic, transcriptomic, and 419 functional changes, depending on the specific environmental stimulus [7,8], including those related to ageing and disease $[10,56]$. Here, we observed that application of various oxysterols, in the same 421 proportions as those identified in early and late-stage $\mathrm{AD}$ brain, distinctly altered astrocyte morphology after $24 \mathrm{~h}$ treatment. In particular, astrocytes adopted a more stellate appearance with many long and branched processes evident. In support of these data, $7 \beta-\mathrm{OHC}$ has previously been shown to induce morphological changes, characterized by process elongation, in cultured reactive astrocytes [41]. An increase in the number of processes is considered a reliable feature of reactivity, demonstrated in several cell culture models of reactive astrocytes $[57,58]$.

Reactive astrocytes were conventionally identified by GFAP immunoreactivity; however, it is noteworthy that different stimuli can lead to similar degrees of GFAP upregulation while causing substantially different changes in transcriptome profile and cell functions [7]. 430 Furthermore, unlike brain astrocytes, most astrocytes in culture are GFAP reactive as a result of the 431 in vitro conditions, the absence of their usual environment, and loss of inhibitory influences of 432 neurons [41]. Astrocytes treated with Early or Late AD oxysterol mixtures up to $24 \mathrm{~h}$ showed a 433 significant but mild increase in GFAP protein levels despite the extensive morphological changes 
observed. The morphological changes we observed in response to oxysterol mixtures were, however,

435 accompanied by a marked upregulation of Len2 and SerpinA3N, both proteins identified as "pan reactive" astrocytes markers [8], as well as by increased release of Lcn2 into culture medium. Lcn2, also known as neutrophil gelatinase-associated lipocalin (NGAL), is an acute phase protein with several roles [14] that is secreted by different kind of cells, including reactive astrocytes [16, 17]. It can act as an autocrine mediator of reactive astrogliosis, since it is able to induce morphological changes in primary astrocytes [57]. In addition, several studies showed that Len2 exerts synaptotoxic 441 and neurotoxic effects, affecting neuronal viability, dendritic spine density and morphology, and ultimately cognitive functions $[17,49,52,53]$. SerpinA3N, also known as alpha1-antichymotrypsin, is a protease inhibitor involved in the acute phase response, that is produced in various tissues including the brain, with reactive and aging astrocytes being the main producers of Lcn 2 in the brain $[8,56]$. Immunohistochemical analysis of AD brain samples showed that SerpinA3N co-localizes with amyloid plaques, reactive microglia and astrocytes [59]. Moreover, an increase in tau phosphorylation has been observed both in mice overexpressing SerpinA3N and in primary neurons treated with recombinant Lcn2, the latter also showing neurite degeneration and apoptosis [60].

It is well known that in many cases astrocyte activation is accompanied by an increase in the release of several soluble factors, including reactive oxygen species and cytokines [11, 48]. Interestingly, 7 $\alpha, 25$-dihydroxycholesterol has been shown to inhibit the lipopolysaccharide (LPS)induced release of pro-inflammatory cytokines including IL-17/tumor necrosis factor- $\alpha$ (TNF- $\alpha$ ) in 453 cultured human astrocytes [61]. Other oxysterols (e.g. 27-OHC) have been demonstrated to decrease LPS-induced expression of IL-6 and TNF- $\alpha$ mRNA in mouse primary glial cells [62]. However, there are no data on how oxysterols directly affect astrocyte secretion profile. Our cytokine array analysis showed that oxysterol treatment significantly increases the release of a wide range of cytokines and chemokines from primary astrocytes after $24 \mathrm{~h}$. Interestingly, this analysis highlighted a differential impact of the two oxysterol mixtures: the majority of the significant increases were observed in ACM 
from astrocytes treated with the mixture that mimics late AD brain composition. Among the released mediators, inflammatory cytokines (IL-1 $\alpha$, IL-1 $\beta$, and IL-17), adhesion molecules (sICAM-1), 461 growth factors and chemokines (IL-5, IL-7, IL-16, G-CSF, CCL1, CCL12, and CXCL9), 462 immunoregulatory cytokines (IL-5 and IL-13), and anti-inflammatory cytokines (IL-10) were 463 elevated. In particular, IL-1 $\beta$ and sICAM-1 were the most significantly increased. Astrocytes are both 464 targets and effectors of cytokines and many other mediators that affect not only their immune and 465 inflammatory cell functions but also their synapse-directed and neuronal functions [48]. IL-1 $\beta$ has 466 been previously shown to induce reactive astrogliosis [10], to be released by reactive astrocytes and astrocytes from 5xFAD AD mouse model $[63,64]$, and to contribute to astrocyte-mediated neuronal death [65]; moreover, its levels are increased in $\mathrm{AD}$ brains $[27,66]$.

Using gene ontology classification, proteins involved in extracellular matrix modification and adhesion were identified as the gene class most represented in the transcriptome of reactive astrocytes [8]. ICAM-1 is a transmembrane glycoprotein belonging to the immunoglobulin family of adhesion molecules, expressed not only by endothelial and immune system cells [67] but also by astrocytes [8]. sICAM-1 is released by proteolytic cleavage of the transmembrane protein $[64,68]$. Several studies have shown that the addition of sICAM-1 to different in vitro models activates proinflammatory cascades, including in astrocytes, and inflammatory mediators can in turn favour its release $[69,70]$. Furthermore, sICAM-1 levels are increased in AD cerebrospinal fluid and correlate with various $\mathrm{AD}$ markers including total tau, phosphorylated tau, and cortical thinning $[71,72]$. The data presented here provide the first evidence for a direct impact of oxysterols on astrocyte morphology, markers of reactivity, and secretion profile, and strongly suggest that oxysterol mixtures that mimic oxysterol composition in $\mathrm{AD}$ brain induce astrocyte reactivity.

The functional implications of astrocyte reactivity are various and very context-dependent. 482 For instance, astrocytes have been shown to mediate $A \beta$-induced neurotoxicity and tau 483 phosphorylation, as well as to induce a decrease of synaptic markers; interestingly, the release of pro- 
inflammatory cytokines from astrocytes seems to play a key role in some of these processes $[11,73]$.

485 To investigate the consequences of oxysterol-mediated astrocytic reactivity on neurons, we exposed neurons to astrocytes that had previously been challenged with oxysterols. Our data indicated that neurons co-cultured with pre-treated astrocytes are characterized by a significant reduction in PSD95 protein levels and a clear increase in cleaved caspase-3 protein levels, suggesting that oxysterolinduced reactivity is detrimental for neuronal health. Importantly, similar changes were obtained when neuronal cultures were directly treated with the Late AD mixture in the absence of astrocytes, confirming that oxysterols have a direct and negative effect on neuron health. PSD95 is a scaffold protein abundant in the postsynaptic density (PSD), that anchors N-methyl-D-aspartate receptor (NMDAR) as well as other proteins (e.g potassium channels, cell adhesion molecules) to the PSD. It is also involved in the assembly of specific signaling proteins, that act downstream of NMDAR [74]. PSD95 is recognized to play an important role in synaptic plasticity and memory, and in-keeping with this its levels decrease in $\mathrm{AD}$ [5]. To the best of our knowledge, no studies have yet assessed the specific actions of oxysterol-treated astrocytes on PSD95 levels, however various mediators released by oxysterol-treated astrocytes have previously been shown to affect synapses, including Lcn2 and IL- $1 \alpha / \beta[52,75,76]$. Indeed, the binding of cytokines and chemokines to their receptors leads to the activation of diverse signaling pathways [77], some of which are involved in PSD95 regulation [78].

Concerning oxysterols, it has been demonstrated that 27-OHC reduces dendritic spine density and

PSD95 levels in primary mouse hippocampal neurons [33], whereas 24-OHC affects synaptic involved in the observed PSD95 reduction, even if their role needs further investigation. Moreover, the ability of oxysterols to alter membrane structure could affect transmembrane protein localization and function, thus modifying the integrity of the PSD structure $[80,81]$. The increase in the levels of cleaved caspase-3 fragment further indicates that oxysterol-activated astrocytes are detrimental to neurons. Besides being an effector of apoptosis, caspase-3 was demonstrated to play various non- 
apoptotic roles in neurons involved in both physiological processes and neurodegenerative diseases

511 [51]. For instance, it is involved in the pathological cleavage of tau protein [82] and its activation 512 correlates with dendritic spine loss and cognitive decline in the absence of neuronal cell death [83]. 513 Interestingly, it has previously been shown that increase in neuronal cleaved caspase-3 levels in 514 response to $\mathrm{A} \beta$ is exacerbated in the presence of reactive astrocytes, and that increased caspase- 3 515 activity is reduced upon pretreatment with an anti-inflammatory molecule in vitro [11] and in vivo 516 [84]. These and other data highlight that neuronal caspase-3 can be activated by extracellular 517 inflammatory mediators. Caspase-3 can also be activated by oxysterols, mainly those oxidized at C7 518 position but also others [33, 50, 85-87].

In $\mathrm{AD}$, synaptic loss is the parameter that best correlates with dementia [88, 89]; therefore, elucidating how synapses are lost is of great importance. This can be examined in vitro by monitoring dendritic spine density and/or neurite complexity. The direct effect of oxysterols on synaptic health has been partially investigated as stated above, but we were interested in assessing how oxysterolactivated astrocytes affect synapses. Our results indicate that ACM from oxysterol-activated astrocytes is synaptotoxic, as shown by significant decreases in the number of dendritic spines and reduced complexity of the neurites in primary neurons, as well as by PSD95 reduction as described above. Because Lcn2 is secreted upon oxysterol-induced astrocyte activation and has proven to be neurotoxic $[17,53]$, we tested whether we could revert the synaptotoxic effect of reactive astrocytes by suppressing Lcn2 expression and therefore reducing its secretion. Our results clearly demonstrate that dendritic spine density and neurites architecture are better preserved when Lcn2 expression is silenced prior to treating astrocytes with oxysterols. These data reveal a major role for Lcn2 in mediating the synaptotoxic effect of oxysterol-treated astrocytes. These findings extend previous 532 studies showing that Len2 affects dendritic spine density and morphology [52], as well as contributing to hippocampal damage and cognitive impairment in a mouse model of vascular dementia [49]. 
536 Overall, these results describe the complexity of oxysterol effects on astrocytes and neurons. Of 537 interest was our observation that astrocytes respond most strongly to oxysterol mixture representative 538 of late $\mathrm{AD}$ brain in comparison to early $\mathrm{AD}$-mimicking mixture. These data add to an emerging 539 consensus that astrocyte responses are differentially altered at specific disease stages in response to 540 various changes in the local environment. Indeed, we demonstrated that oxysterols induce a clear 541 morphological change in astrocytes, that is accompanied by an increase in some reactive astrocyte 542 markers and the release of several mediators. We also demonstrated that oxysterols are detrimental 543 to neuronal health but do not cause overt neurotoxicity. Importantly, we have shown that oxysterol544 activated astrocytes induce synaptotoxicity that is mediated by Lcn2. This study thus reveals new 545 aspects of brain oxysterol effects on astrocytes and neurons, confirming their potential ability to 546 contribute to AD pathogenesis, and providing support for further investigations into a potential role 547 for Lcn2 as a novel therapeutic target in AD.

\section{ABBREVIATIONS}

$\alpha$-EPOX: $\quad 5 \alpha, 6 \alpha$-epoxycholesterol; $\quad \beta$-EPOX: $\quad 5 \beta, 6 \beta$-epoxycholesterol; $\quad 24-\mathrm{OHC}: \quad 24-$ 551 hydroxycholesterol; 27-OHC: 27-hydroxycholesterol; 7-KC: 7-ketocholesterol; 7 $\alpha$-OHC: $7 \alpha-$ hydroxycholesterol; 7 $\beta$-OHC: 7 $\beta$-hydroxycholesterol; A $\beta$ : Amyloid- $\beta$; ACM: Astrocyte conditioned media; AD: Alzheimer's disease; ApoE: Apolipoprotein E; BBB: Blood-brain barrier; CCL: C-C motif chemokine; CXCL: C-X-C motif chemokine; CYP46A1: Cholesterol 24-hydroxylase; DIV: 555 Days in vitro; GC-MS: Gas chromatography-mass spectrometry; G-CSF: Granulocyte colonystimulating factor; GFAP: Glial fibrillary acidic protein; Iba1: Ionized calcium binding adaptor molecule 1; IL: Interleukin; Lcn2: Lipocalin-2; LDH: Lactate dehydrogenase, LPS: Lipopolysaccharide; NFTs: Neurofibrillary tangles; NGAL: Neutrophil gelatinase-associated 559 lipocalin; NMDAR: N-methyl-D-aspartate receptor; PSD: Postsynaptic density; PSD95: Postsynaptic 
560 density protein 95; SerpinA3N: Serine protease inhibitor A3N; sICAM-1: Soluble intercellular 561 adhesion molecule-1; siRNA: Small interfering RNA; TNF- $\alpha$ : Tumor necrosis factor- $\alpha$.

\section{DECLARATIONS}

Ethics approval and consent to participate

565 All animal procedures were in accordance with the European Communities Council Directive 566 (86/609/EEC and 2010/63/EU), the UK Animals (Scientific Procedures) Act 1986, and the Italian 567 Law for Care and Use of Experimental Animals (26/2016), with agreement from both the King's 568 College London (Denmark Hill) Animal Welfare and Ethical Review Board and the Ethical 569 Committee of the University of Turin.

572 Not applicable

\section{Availability of data and materials}

575 All data generated or analysed during this study are included in this published article [and its 576 supplementary information files].

\section{Competing interests}

579 The authors declare that they have no competing interests. 


\section{Funding}

582 This project was supported by funding from Alzheimer's Research UK (ARUK-RF2014-2, ARUK583 EG2013-B1, ARUK-PG2019A-004), the University of Turin (RILO 2019), and Compagnia di San 584 Paolo Foundation (CST0167048). ES was supported by Adriano Buzzati-Traverso Foundation.

\section{Author's contributions}

ES, VC and BGPN designed and performed experiments. ES and BGPN analysed samples and performed the statistical analysis. VL and CC performed GC-MS analysis and analysed data. ES and BGPN wrote the manuscript. $\mathrm{AB}, \mathrm{WN}$, and GL revised the manuscript. PG, WN, BGPN, and GL obtained the funding. ES, BGPN, GT and SG prepared the figures. All authors read and approved the

591 final manuscript.

\section{Acknowledgements}

Not applicable

\section{REFERENCES}

1. Prince MJ WA, Guerchet MM, Ali GC, Wu YT, Prina AM, Alzheimer's Disease International.

598 World Alzheimer Report 2015: The Global Impact of Dementia: An Analysis of Prevalence, 599 Incidence, Cost and Trends. Alzheimer’s Disease International; 2015.

$600 \quad$ 2. Lane CA, Hardy J, Schott JM. Alzheimer's disease. Eur J Neurol. 2018;25:59-70.

601 3. Serrano-Pozo A, Frosch MP, Masliah E, Hyman BT. Neuropathological alterations in 602 Alzheimer disease. Cold Spring Harb Perspect Med. 2011;1:a006189. 
603 4. Perez-Nievas BG, Serrano-Pozo A. Deciphering the Astrocyte Reaction in Alzheimer's 604 Disease. Front Aging Neurosci. 2018;10:114.

605 5. Perez-Nievas BG, Stein TD, Tai HC, Dols-Icardo O, Scotton TC, Barroeta-Espar I, et al. 606 Dissecting phenotypic traits linked to human resilience to Alzheimer's pathology. Brain. $607 \quad 2013 ; 136: 2510-26$.

608 6. Vehmas AK, Kawas CH, Stewart WF, Troncoso JC. Immune reactive cells in senile plaques 609 and cognitive decline in Alzheimer's disease. Neurobiol Aging. 2003;24:321-31.

610 7. Anderson MA, Ao Y, Sofroniew MV. Heterogeneity of reactive astrocytes. Neurosci Lett. $611 \quad 2014 ; 565: 23-9$.

612 8. Zamanian JL, Xu L, Foo LC, Nouri N, Zhou L, Giffard RG, et al. Genomic analysis of reactive 613 astrogliosis. J Neurosci. 2012;32:6391-410.

614 9. Iram T, Trudler D, Kain D, Kanner S, Galron R, Vassar R, et al. Astrocytes from old 615 Alzheimer's disease mice are impaired in Abeta uptake and in neuroprotection. Neurobiol Dis. $616 \quad 2016 ; 96: 84-94$.

617 10. Liddelow SA, Guttenplan KA, Clarke LE, Bennett FC, Bohlen CJ, Schirmer L, et al. 618 Neurotoxic reactive astrocytes are induced by activated microglia. Nature. 2017;541:481-7.

619 11. Garwood CJ, Pooler AM, Atherton J, Hanger DP, Noble W. Astrocytes are important 620 mediators of Abeta-induced neurotoxicity and tau phosphorylation in primary culture. Cell Death Dis. $621 \quad 2011 ; 2: \mathrm{e} 167$.

622 12. Cavanagh C, Tse YC, Nguyen HB, Krantic S, Breitner JC, Quirion R, et al. Inhibiting tumor 623 necrosis factor-alpha before amyloidosis prevents synaptic deficits in an Alzheimer's disease model. 624 Neurobiol Aging. 2016;47:41-9.

625 13. Lian H, Yang L, Cole A, Sun L, Chiang AC, Fowler SW, et al. NFkappaB-activated astroglial 626 release of complement C3 compromises neuronal morphology and function associated with 627 Alzheimer's disease. Neuron. 2015;85:101-15. 
628 14. Jha MK, Lee S, Park DH, Kook H, Park KG, Lee IK, et al. Diverse functional roles of 629 lipocalin-2 in the central nervous system. Neurosci Biobehav Rev. 2015;49:135-56.

630 15. Naude PJ, Nyakas C, Eiden LE, Ait-Ali D, van der Heide R, Engelborghs S, et al. Lipocalin 631 2: novel component of proinflammatory signaling in Alzheimer's disease. FASEB J. 2012;26:281163223.

633 16. Ferreira AC, Da Mesquita S, Sousa JC, Correia-Neves M, Sousa N, Palha JA, et al. From the 634 periphery to the brain: Lipocalin-2, a friend or foe? Prog Neurobiol. 2015;131:120-36.

635 17. Bi F, Huang C, Tong J, Qiu G, Huang B, Wu Q, et al. Reactive astrocytes secrete lcn2 to 636 promote neuron death. Proc Natl Acad Sci U S A. 2013;110:4069-74.

637 18. Alzheimer A, Stelzmann RA, Schnitzlein HN, Murtagh FR. An English translation of 638 Alzheimer's 1907 paper, "Uber eine eigenartige Erkankung der Hirnrinde". Clin Anat. 1995;8:42963931.

640 19. Corder EH, Saunders AM, Strittmatter WJ, Schmechel DE, Gaskell PC, Small GW, et al. 641 Gene dose of apolipoprotein E type 4 allele and the risk of Alzheimer's disease in late onset families. 642 Science. 1993;261:921-3.

643 20. Di Paolo G, Kim TW. Linking lipids to Alzheimer's disease: cholesterol and beyond. Nat Rev 644 Neurosci. 2011;12:284-96.

645 21. Wood WG, Li L, Muller WE, Eckert GP. Cholesterol as a causative factor in Alzheimer's 646 disease: a debatable hypothesis. J Neurochem. 2014;129:559-72.

647 22. Gamba P, Testa G, Gargiulo S, Staurenghi E, Poli G, Leonarduzzi G. Oxidized cholesterol as 648 the driving force behind the development of Alzheimer's disease. Front Aging Neurosci. 2015;7.

649 23. Dason JS, Smith AJ, Marin L, Charlton MP. Cholesterol and F-actin are required for clustering 650 of recycling synaptic vesicle proteins in the presynaptic plasma membrane. J Physiol. 2014;592:62165133. 
652 24. van Deijk AF, Camargo N, Timmerman J, Heistek T, Brouwers JF, Mogavero F, et al. 653 Astrocyte lipid metabolism is critical for synapse development and function in vivo. Glia. $654 \quad 2017 ; 65: 670-82$.

655 25. Lutjohann D, Breuer O, Ahlborg G, Nennesmo I, Siden A, Diczfalusy U, et al. Cholesterol 656 homeostasis in human brain: evidence for an age-dependent flux of 24S-hydroxycholesterol from the 657 brain into the circulation. Proc Natl Acad Sci U S A. 1996;93:9799-804.

658 26. Iuliano L, Crick PJ, Zerbinati C, Tritapepe L, Abdel-Khalik J, Poirot M, et al. Cholesterol 659 metabolites exported from human brain. Steroids. 2015;99:189-93.

660 27. Testa G, Staurenghi E, Zerbinati C, Gargiulo S, Iuliano L, Giaccone G, et al. Changes in brain 661 oxysterols at different stages of Alzheimer's disease: Their involvement in neuroinflammation. Redox 662 Biol. 2016;10:24-33.

663 28. Mutemberezi V, Guillemot-Legris O, Muccioli GG. Oxysterols: From cholesterol metabolites 664 to key mediators. Prog Lipid Res. 2016;64:152-69.

665 29. Testa G, Gamba P, Badilli U, Gargiulo S, Maina M, Guina T, et al. Loading into nanoparticles 666 improves quercetin's efficacy in preventing neuroinflammation induced by oxysterols. PLoS One. $667 \quad 2014 ; 9: \mathrm{e} 96795$.

668 30. Zhang X, Xi Y, Yu H, An Y, Wang Y, Tao L, et al. 27-hydroxycholesterol promotes Abeta 669 accumulation via altering Abeta metabolism in mild cognitive impairment patients and APP/PS1 670 mice. Brain Pathol. 2019;29:558-73.

671 31. Gamba P, Leonarduzzi G, Tamagno E, Guglielmotto M, Testa G, Sottero B, et al. Interaction 672 between 24-hydroxycholesterol, oxidative stress, and amyloid-beta in amplifying neuronal damage 673 in Alzheimer's disease: three partners in crime. Aging Cell. 2011;10:403-17.

674 32. Marwarha G, Dasari B, Prasanthi JR, Schommer J, Ghribi O. Leptin reduces the accumulation 675 of Abeta and phosphorylated tau induced by 27-hydroxycholesterol in rabbit organotypic slices. J 676 Alzheimers Dis. 2010;19:1007-19. 
33. Merino-Serrais P, Loera-Valencia R, Rodriguez-Rodriguez P, Parrado-Fernandez C, Ismail

MA, Maioli S, et al. 27-Hydroxycholesterol Induces Aberrant Morphology and Synaptic Dysfunction in Hippocampal Neurons. Cereb Cortex. 2019;29:429-46.

34. Jang ER, Lee CS. 7-ketocholesterol induces apoptosis in differentiated PC12 cells via reactive oxygen species-dependent activation of NF-kappaB and Akt pathways. Neurochem Int. 2011;58:529.

35. Nury T, Samadi M, Zarrouk A, Riedinger JM, Lizard G. Improved synthesis and in vitro evaluation of the cytotoxic profile of oxysterols oxidized at C4 (4alpha- and 4betahydroxycholesterol) and C7 (7-ketocholesterol, 7alpha- and 7beta-hydroxycholesterol) on cells of the central nervous system. Eur J Med Chem. 2013;70:558-67.

36. An Y, Zhang DD, Yu HL, Ma WW, Lu YH, Liu QR, et al. 27-Hydroxycholesterol regulates cholesterol synthesis and transport in C6 glioma cells. Neurotoxicology. 2017;59:88-97.

37. Abildayeva K, Jansen PJ, Hirsch-Reinshagen V, Bloks VW, Bakker AH, Ramaekers FC, et al. 24(S)-hydroxycholesterol participates in a liver X receptor-controlled pathway in astrocytes that 691 regulates apolipoprotein E-mediated cholesterol efflux. J Biol Chem. 2006;281:12799-808.

692 38. Zhang DD, Yu HL, Ma WW, Liu QR, Han J, Wang H, et al. 27-Hydroxycholesterol 693 contributes to disruptive effects on learning and memory by modulating cholesterol metabolism in 694 the rat brain. Neuroscience. 2015;300:163-73.

695 39. Ma WW, Li CQ, Yu HL, Zhang DD, Xi YD, Han J, et al. The oxysterol 27-hydroxycholesterol 696 increases oxidative stress and regulate Nrf2 signaling pathway in astrocyte cells. Neurochem Res. $697 \quad 2015 ; 40: 758-66$.

698 40. Cigliano L, Spagnuolo MS, Napolitano G, Iannotta L, Fasciolo G, Barone D, et al. 24S699 hydroxycholesterol affects redox homeostasis in human glial U-87MG cells. Mol Cell Endocrinol. 700 2019;486:25-33. 
701 41. Bochelen D, Langley K, Adamczyk M, Kupferberg A, Hor F, Vincendon G, et al. 7beta702 hydroxysterol is cytotoxic to neonatal rat astrocytes in primary culture when cAMP levels are 703 increased. J Neurosci Res. 2000;62:99-111.

704 42. Schildge S, Bohrer C, Beck K, Schachtrup C. Isolation and culture of mouse cortical 705 astrocytes. J Vis Exp. 2013.

706 43. Hudry E, Wu HY, Arbel-Ornath M, Hashimoto T, Matsouaka R, Fan Z, et al. Inhibition of the 707 NFAT pathway alleviates amyloid beta neurotoxicity in a mouse model of Alzheimer's disease. J 708 Neurosci. 2012;32:3176-92.

709 44. Leoni V, Strittmatter L, Zorzi G, Zibordi F, Dusi S, Garavaglia B, et al. Metabolic 710 consequences of mitochondrial coenzyme A deficiency in patients with PANK2 mutations. Mol 711 Genet Metab. 2012;105:463-71.

712 45. Livak KJ, Schmittgen TD. Analysis of relative gene expression data using real-time 713 quantitative PCR and the 2(-Delta Delta C(T)) Method. Methods. 2001;25:402-8.

714 46. Sovrea AS, Bosca AB. Astrocytes reassessment - an evolving concept part one: embryology, 715 biology, morphology and reactivity. J Mol Psychiatry. 2013;1:18.

716 47. Liddelow SA, Barres BA. Reactive Astrocytes: Production, Function, and Therapeutic 717 Potential. Immunity. 2017;46:957-67.

718 48. Sofroniew MV. Multiple roles for astrocytes as effectors of cytokines and inflammatory 719 mediators. Neuroscientist. 2014;20:160-72.

720 49. Kim JH, Ko PW, Lee HW, Jeong JY, Lee MG, Kim JH, et al. Astrocyte-derived lipocalin-2 721 mediates hippocampal damage and cognitive deficits in experimental models of vascular dementia. 722 Glia. 2017;65:1471-90.

723 50. Lordan S, Mackrill JJ, O'Brien NM. Oxysterols and mechanisms of apoptotic signaling: 724 implications in the pathology of degenerative diseases. J Nutr Biochem. 2009;20:321-36.

725 51. D'Amelio M, Sheng M, Cecconi F. Caspase-3 in the central nervous system: beyond 726 apoptosis. Trends Neurosci. 2012;35:700-9. 
52. Mucha M, Skrzypiec AE, Schiavon E, Attwood BK, Kucerova E, Pawlak R. Lipocalin-2 controls neuronal excitability and anxiety by regulating dendritic spine formation and maturation. Proc Natl Acad Sci U S A. 2011;108:18436-41.

730 53. Lee S, Lee WH, Lee MS, Mori K, Suk K. Regulation by lipocalin-2 of neuronal cell death, 731 migration, and morphology. J Neurosci Res. 2012;90:540-50.

732 54. Karch CM, Goate AM. Alzheimer's disease risk genes and mechanisms of disease 733 pathogenesis. Biol Psychiatry. 2015;77:43-51.

734 55. Serrano-Pozo A, Mielke ML, Gomez-Isla T, Betensky RA, Growdon JH, Frosch MP, et al. 735 Reactive glia not only associates with plaques but also parallels tangles in Alzheimer's disease. Am J 736 Pathol. 2011;179:1373-84.

737 56. Boisvert MM, Erikson GA, Shokhirev MN, Allen NJ. The Aging Astrocyte Transcriptome 738 from Multiple Regions of the Mouse Brain. Cell Rep. 2018;22:269-85.

739 57. Lee S, Park JY, Lee WH, Kim H, Park HC, Mori K, et al. Lipocalin-2 is an autocrine mediator 740 of reactive astrocytosis. J Neurosci. 2009;29:234-49.

741 58. Holtje M, Hoffmann A, Hofmann F, Mucke C, Grosse G, Van Rooijen N, et al. Role of Rho 742 GTPase in astrocyte morphology and migratory response during in vitro wound healing. J $743 \quad$ Neurochem. 2005;95:1237-48.

744 59. Styren SD, Kamboh MI, DeKosky ST. Expression of differential immune factors in temporal 745 cortex and cerebellum: the role of alpha-1-antichymotrypsin, apolipoprotein E, and reactive glia in 746 the progression of Alzheimer's disease. J Comp Neurol. 1998;396:511-20.

747 60. Padmanabhan J, Levy M, Dickson DW, Potter H. Alpha1-antichymotrypsin, an inflammatory 748 protein overexpressed in Alzheimer's disease brain, induces tau phosphorylation in neurons. Brain. $749 \quad 2006 ; 129: 3020-34$.

750 61. Rutkowska A, Shimshek DR, Sailer AW, Dev KK. EBI2 regulates pro-inflammatory 751 signalling and cytokine release in astrocytes. Neuropharmacology. 2018;133:121-8. 
62. Mutemberezi V, Buisseret B, Masquelier J, Guillemot-Legris O, Alhouayek M, Muccioli GG.

753 Oxysterol levels and metabolism in the course of neuroinflammation: insights from in vitro and in 754 vivo models. J Neuroinflammation. 2018;15:74.

63. van Gijsel-Bonnello M, Baranger K, Benech P, Rivera S, Khrestchatisky M, de Reggi M, et al. Metabolic changes and inflammation in cultured astrocytes from the 5xFAD mouse model of Alzheimer's disease: Alleviation by pantethine. PLoS One. 2017;12:e0175369.

64. Choi SS, Lee HJ, Lim I, Satoh J, Kim SU. Human astrocytes: secretome profiles of cytokines and chemokines. PLoS One. 2014;9:e92325.

65. Sharma V, Mishra M, Ghosh S, Tewari R, Basu A, Seth P, et al. Modulation of interleukinlbeta mediated inflammatory response in human astrocytes by flavonoids: implications in neuroprotection. Brain Res Bull. 2007;73:55-63.

66. Morales I, Guzman-Martinez L, Cerda-Troncoso C, Farias GA, Maccioni RB. Neuroinflammation in the pathogenesis of Alzheimer's disease. A rational framework for the search of novel therapeutic approaches. Front Cell Neurosci. 2014;8:112.

67. Hubbard AK, Rothlein R. Intercellular adhesion molecule-1 (ICAM-1) expression and cell signaling cascades. Free Radic Biol Med. 2000;28:1379-86.

68. Lyons PD, Benveniste EN. Cleavage of membrane-associated ICAM-1 from astrocytes: involvement of a metalloprotease. Glia. 1998;22:103-12.

69. Otto VI, Gloor SM, Frentzel S, Gilli U, Ammann E, Hein AE, et al. The production of macrophage inflammatory protein-2 induced by soluble intercellular adhesion molecule-1 in mouse astrocytes is mediated by src tyrosine kinases and p42/44 mitogen-activated protein kinase. J 773 Neurochem. 2002;80:824-34.

774 70. Lawson C, Wolf S. ICAM-1 signaling in endothelial cells. Pharmacol Rep. 2009;61(1):22-32. 775 71. Trombetta BA, Carlyle BC, Koenig AM, Shaw LM, Trojanowski JQ, Wolk DA, et al. The 776 technical reliability and biotemporal stability of cerebrospinal fluid biomarkers for profiling multiple 777 pathophysiologies in Alzheimer's disease. PLoS One. 2018;13:e0193707. 

biomarkers of neuroinflammation and cerebrovascular dysfunction in early Alzheimer disease. Neurology. 2018;91:e867-e77.

73. Allaman I, Gavillet M, Belanger M, Laroche T, Viertl D, Lashuel HA, et al. Amyloid-beta aggregates cause alterations of astrocytic metabolic phenotype: impact on neuronal viability. $\mathbf{J}$ Neurosci. 2010;30:3326-38.

74. Sheng M. The postsynaptic NMDA-receptor--PSD-95 signaling complex in excitatory synapses of the brain. J Cell Sci. 2001;114:1251.

75. Prieto GA, Cotman CW. Cytokines and cytokine networks target neurons to modulate longterm potentiation. Cytokine Growth Factor Rev. 2017;34:27-33.

76. Khairova RA, Machado-Vieira R, Du J, Manji HK. A potential role for pro-inflammatory cytokines in regulating synaptic plasticity in major depressive disorder. Int J Neuropsychopharmacol. 2009;12:561-78.

77. Turner MD, Nedjai B, Hurst T, Pennington DJ. Cytokines and chemokines: At the crossroads of cell signalling and inflammatory disease. Biochim Biophys Acta. 2014;1843(11):2563-82.

78. Mao LM, Wang JQ. Synaptically Localized Mitogen-Activated Protein Kinases: Local Substrates and Regulation. Mol Neurobiol. 2016;53:6309-15.

79. Paul SM, Doherty JJ, Robichaud AJ, Belfort GM, Chow BY, Hammond RS, et al. The major brain cholesterol metabolite 24(S)-hydroxycholesterol is a potent allosteric modulator of N-methylD-aspartate receptors. J Neurosci. 2013;33:17290-300.

80. Kulig W, Cwiklik L, Jurkiewicz P, Rog T, Vattulainen I. Cholesterol oxidation products and their biological importance. Chem Phys Lipids. 2016;199:144-60.

800 81. Dias IH, Borah K, Amin B, Griffiths HR, Sassi K, Lizard G, et al. Localisation of oxysterols 801 at the sub-cellular level and in biological fluids. J Steroid Biochem Mol Biol. 2019;193:105426. 
83. D'Amelio M, Cavallucci V, Middei S, Marchetti C, Pacioni S, Ferri A, et al. Caspase-3 triggers early synaptic dysfunction in a mouse model of Alzheimer's disease. Nat Neurosci. 2011;14:69-76. reduces the development of abnormal tau species in models of Alzheimer's disease. FASEB J. 2009;23:739-50.

85. Ragot K, Delmas D, Athias A, Nury T, Baarine M, Lizard G. alpha-Tocopherol impairs 7ketocholesterol-induced caspase-3-dependent apoptosis involving GSK-3 activation and Mcl-1 degradation on 158N murine oligodendrocytes. Chem Phys Lipids. 2011;164:469-78. 24(S)-hydroxycholesterol-induced necroptosis-like cell death and apoptosis. J Steroid Biochem Mol Biol. 2017;169:69-76.

87. Kim A, Nam YJ, Lee CS. Taxifolin reduces the cholesterol oxidation product-induced neuronal apoptosis by suppressing the Akt and NF-kappaB activation-mediated cell death. Brain Res Bull. 2017;134:63-71.

88. DeKosky ST, Scheff SW. Synapse loss in frontal cortex biopsies in Alzheimer's disease: correlation with cognitive severity. Ann Neurol. 1990;27:457-64.

89. Crimins JL, Pooler A, Polydoro M, Luebke JI, Spires-Jones TL. The intersection of amyloid beta and tau in glutamatergic synaptic dysfunction and collapse in Alzheimer's disease. Ageing Res Rev. 2013;12:757-63.

\section{FIGURE LEGENDS}

Fig. 1 Dose-response experiments to test the effect of the oxysterol mixtures on astrocyte oxysterol mixture $(1,5$, or $10 \mu \mathrm{M})$, or vehicle (ethanol) for $24 \mathrm{~h}$. (A) The bar graph shows the lactate dehydrogenase $(\mathrm{LDH})$ release from treated astrocytes. Values are the proportion of LDH released 
into medium relative to total LDH in lysed cells normalized to values for control media. Data are expressed as mean \pm SD from three different experiments ( $n=9$, one-way ANOVA). (B) Astrocyte morphology was examined by immunocytochemistry using a glial fibrillary acidic protein (GFAP) antibody (red) and nuclei were stained with Hoechst 33258 (blue). Representative images from three experiments are shown. Cells were imaged using an LSM800 confocal microscope (Zeiss; 40X objective; scale bar: $100 \mu \mathrm{m})$.

Fig. 2 Oxysterol mixtures induce a clear morphological change in astrocytes. Primary astrocytes were treated with $10 \mu \mathrm{M}$ of the Early or Late $\mathrm{AD}$ oxysterol mixture for up to $24 \mathrm{~h}$. Astrocyte morphology was examined by immunocytochemistry using a glial fibrillary acidic protein (GFAP) antibody (red) and nuclei were stained with Hoechst 33258 (blue). Representative images from three experiments are shown. Cells were imaged using an Eclipse Ti-E Microscope (Nikon; 20X objective; scale bar: $100 \mu \mathrm{m})$.

Fig. 3 Oxysterol mixtures increase the synthesis of pan-reactive astrocyte markers. The glial fibrillary acidic protein (GFAP), serine protease inhibitor A3N (SerpinA3N), and lipocalin-2 (Lcn2) protein levels were determined by Western blotting of lysates from primary astrocytes treated with $10 \mu \mathrm{M}$ of the Early or Late AD oxysterol mixture for up to $24 \mathrm{~h}$. The amounts of proteins of interest were normalized to $\beta$-actin levels in the same sample and are represented as percentage of average control values. Data are expressed as mean values \pm SD of three different experiments $(n=9$, one-way ANOVA). ${ }^{* * * *} \mathrm{P}<0.0001, * * * \mathrm{P}<0.001, * \mathrm{P}<0.05$ vs. control.

\section{Fig. 4 Oxysterol treatment increases the release of lipocalin-2 (Lcn2), cytokines and chemokines}

from astrocytes. Astrocyte cultures were treated with the Early or Late AD oxysterol mixture (10 
$\mu \mathrm{M}$ ) for $24 \mathrm{~h}$. (A) Lcn2 protein levels in astrocyte conditioned media (ACM) were examined by

854 Western blotting. Data are expressed as mean values \pm SD from three different experiments and are 855 shown as percentage change from average control values $(\mathrm{n}=9$, one-way ANOVA). $* * * * \mathrm{P}<0.0001$ 856 vs control. (B) Mouse cytokine profiler antibody arrays were used to detect the amounts of 40 857 mediators in ACM. Representative images of array membranes are shown, highlighted some of the 858 cytokines found to be significantly increased in ACM from treated cells. The bar chart shows the 859 cytokines significantly affected by oxysterol mixture treatments as percentage change from control. 860 Data are expressed as mean values \pm SD from three different experiments ( $n=6$, one-way ANOVA). $* * * \mathrm{P}<0.001, * * \mathrm{P}<0.01$, and $* \mathrm{P}<0.05$ vs control.

Fig. 5 Oxysterol-stimulated astrocytes compromise neuronal health. Primary neurons (13 DIV) were treated with the Late AD oxysterol mixture $(10 \mu \mathrm{M})$ for $24 \mathrm{~h}$ or they were co-cultured for $24 \mathrm{~h}$ with astrocytes grown on cell culture inserts that had been previously treated with the same oxysterol mixture for 12h. (A) Graphical representation of co-culture experiments. (B) The protein levels of the postsynaptic density protein 95 (PSD95), synapsin, and cleaved caspase-3 were determined by Western blotting. Data were normalized to the corresponding $\beta$-actin levels. Data are expressed as mean values \pm SD of three different experiments as percentage change from respective control $(n=9$, Student's t-test). $* * \mathrm{P}<0.01$ and $* \mathrm{P}<0.05$ vs control. medium. Astrocyte cultures were transfected for $6 \mathrm{~h}$ with Lcn 2 or scrambled siRNA and then treated with the Late $\mathrm{AD}$ oxysterol mixture $10 \mu \mathrm{M}$ (Mix) for $12 \mathrm{~h}$. After treatment, the medium was changed and astrocytes were incubated with fresh medium for $24 \mathrm{~h}$. Transient Lcn2 gene knockdown was 
conditioned media (ACM) samples. Data were normalized to the corresponding $\beta$-actin levels. Data are expressed as mean values \pm SD from three different experiments as percentage change from control ( $\mathrm{n}=9$, one-way ANOVA). $* * * * \mathrm{P}<0.0001$, *** $\mathrm{P}<0.001$, and $* \mathrm{P}<0.05$ vs control; \#\#\#\# $\mathrm{P}<0.0001$ vs oxysterol treated. (C) Astrocyte morphology was examined by immunocytochemistry using a glial fibrillary acidic protein (GFAP) antibody (red) and nuclei were stained with Hoechst 33258 (blue). Representative images from three experiments are shown. Cells were imaged using an LSM800 confocal microscope (Zeiss, 40X objective; scale bar: $100 \mu \mathrm{m}$ ).

\section{Fig. 7 Lipocalin-2 (Lcn2) secreted by oxysterol-stimulated astrocytes affects neurite complexity} and decreases dendritic spine density. Primary neurons were incubated for $24 \mathrm{~h}$ with conditioned media from astrocytes transfected for $6 \mathrm{~h}$ with Lcn2 or scrambled siRNA and then treated with the Late $\mathrm{AD}$ oxysterol mixture $10 \mu \mathrm{M}$ for $12 \mathrm{~h}$. After treatment, the medium was changed and astrocytes were incubated with fresh medium for $24 \mathrm{~h}$. High resolution digital images of live neurons were obtained using an Opera-Phenix microscope (Perkin-Elmer). (A) Neurite complexity was analysed using Harmony software. Total and maximum neurite length, number of nodes and extremities were quantified and compared between groups. Representative images are shown (scale bar: $100 \mu \mathrm{m}$ ).

Data are expressed as mean values \pm SD from three different experiments and were obtained from analysis of 20 neurons/condition (one-way ANOVA). ${ }^{*} \mathrm{P}<0.05$ vs control. (B) NeuronStudio software was used for dendritic spine analysis. Spine density was defined as number of spines per micrometer of dendrite length and was obtained from analysis of 20 neurons/condition (one-way ANOVA). Representative images are shown (scale bar: $25 \mu \mathrm{m}$ ). Data are expressed as mean values \pm SD from three different experiments. $* * * \mathrm{P}<0.001$ vs control. 
Supplemental Fig. 1 Evaluation of astrocyte culture purity. Astrocytic cultures were analysed by

904 (A) immunocytochemistry and (B) Western blotting using antibodies against glial fibrillary acidic 905 protein (GFAP, astrocytic marker) and ionized calcium binding adaptor molecule 1 (Iba1, microglial 906 marker). Cells were imaged using an LSM800 confocal microscope (Zeiss, 40X objective; scale bar: $907100 \mu \mathrm{m})$.

908

909 Additional file 2

910 TIFF

911 Supplemental Fig. 2 Validation of lipocalin-2 (Lcn2) silencing efficiency. Astrocyte cultures were 912 transfected for $6 \mathrm{~h}$ with Lcn 2 or scrambled siRNA, then the medium was changed and astrocytes were 913 incubated with fresh medium for 36h. Transient Lcn2 gene knockdown was evaluated by real-time 914 RT-PCR. Data were normalized to the corresponding $\beta$-actin levels. Data are expressed as mean 915 values \pm SD of three different experiments ( $\mathrm{n}=9$, one-way ANOVA). $* * * * \mathrm{P}<0.0001$ vs control.

917 Additional file 3

918 TIFF

919 Supplemental Fig. 3 Oxysterol analyses in astrocyte culture media. Astrocyte cultures were 920 treated for $1,3,12$ or $24 \mathrm{~h}$ with the Early or Late AD oxysterol mixtures $(10 \mu \mathrm{M})$. Astrocyte 921 conditioned media was collected and the amounts of the seven oxysterols present in the mixtures were 922 determined by gas chromatography-mass spectrometry (GC-MS). The graph shows the amounts of oxysterol in media expressed as percentage of the original oxysterol concentrations present in each 
924 mixture. Data shown are averages of the measurements obtained from treatments with Early and Late 925 AD mixtures. Data are expressed as mean values \pm SD from two different experiments $(n=6)$.

$927 \quad$ Additional file 4

928 TIFF

929 Supplemental Fig. 4 Oxysterol mixtures do not directly cause neuron death. Neuronal viability 930 was assessed in neurons treated with the Early or Late AD oxysterol mixture $(10 \mu \mathrm{M})$ for $24 \mathrm{~h}$. The 931 bar graph shows the proportion of lactate dehydrogenase (LDH) released into medium relative to total 932 LDH in lysed cells, normalized to values for control media. Data are expressed as mean values \pm SD 933 from three different experiments ( $\mathrm{n}=12$, one-way ANOVA).

934

935

936

937 


\section{Figures}

A

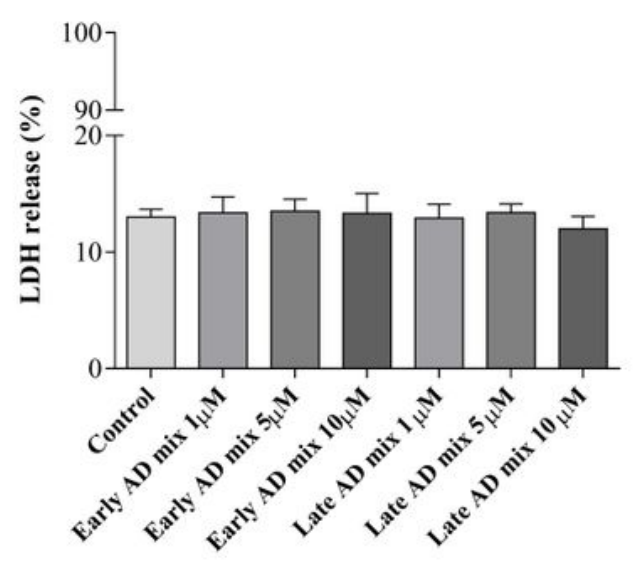

B
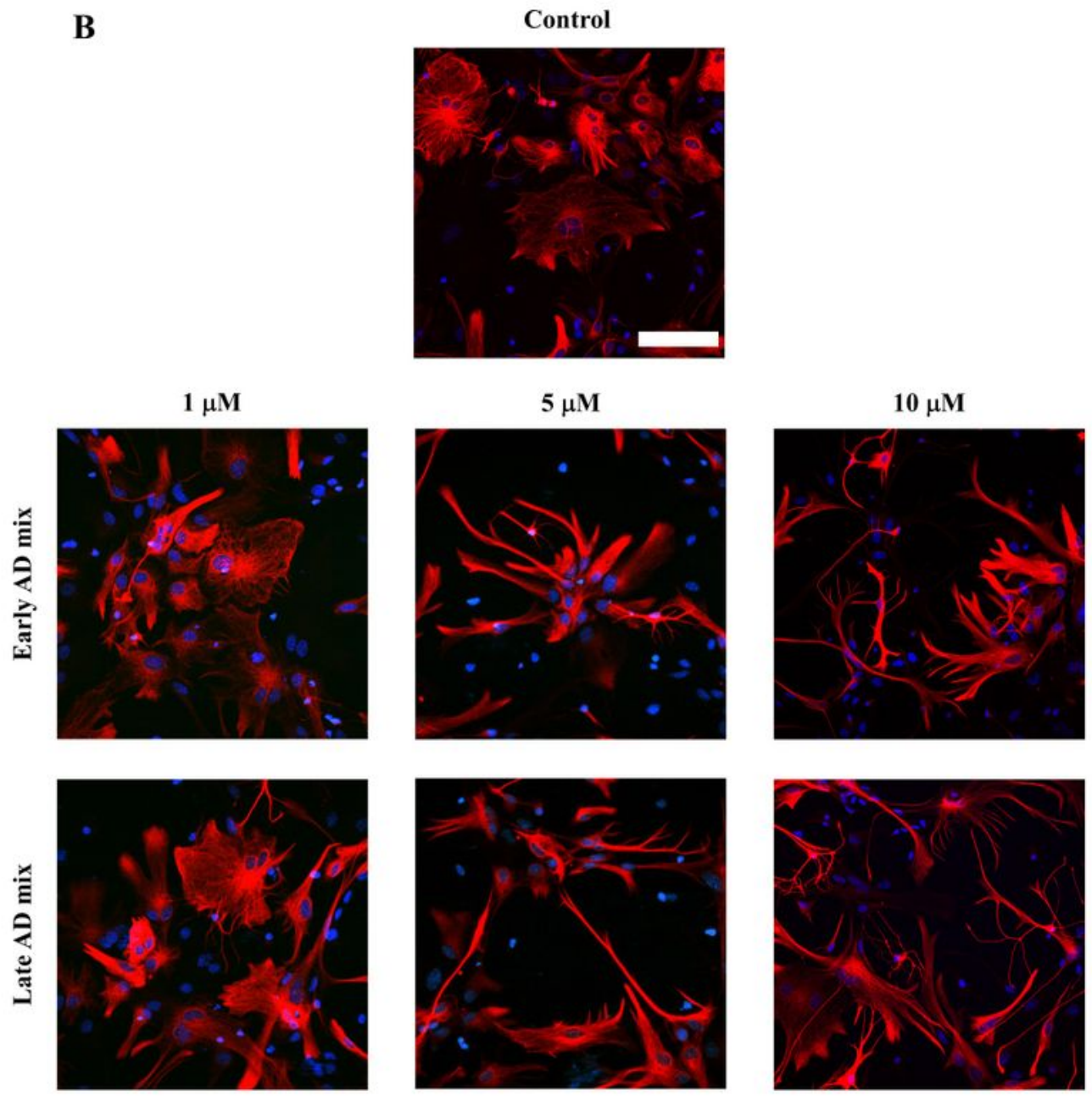

Figure 1

Dose-response experiments to test the effect of the oxysterol mixtures on astrocyte viability and morphology. Primary astrocyte cultures were treated with the Early or Late AD oxysterol mixture $(1,5$, or $10 \varangle \mathrm{M})$, or vehicle (ethanol) for $24 \mathrm{~h}$. (A) The bar graph shows the lactate dehydrogenase (LDH) release 
from treated astrocytes. Values are the proportion of LDH released into medium relative to total LDH in lysed cells normalized to values for control media. Data are expressed as mean \pm SD from three different experiments ( $n=9$, one-way ANOVA). (B) Astrocyte morphology was examined by immunocytochemistry using a glial fibrillary acidic protein (GFAP) antibody (red) and nuclei were stained with Hoechst 33258 (blue). Representative images from three experiments are shown. Cells were imaged using an LSM800 confocal microscope (Zeiss; $40 \mathrm{X}$ objective; scale bar: $100 \mathrm{Dm}$ ).

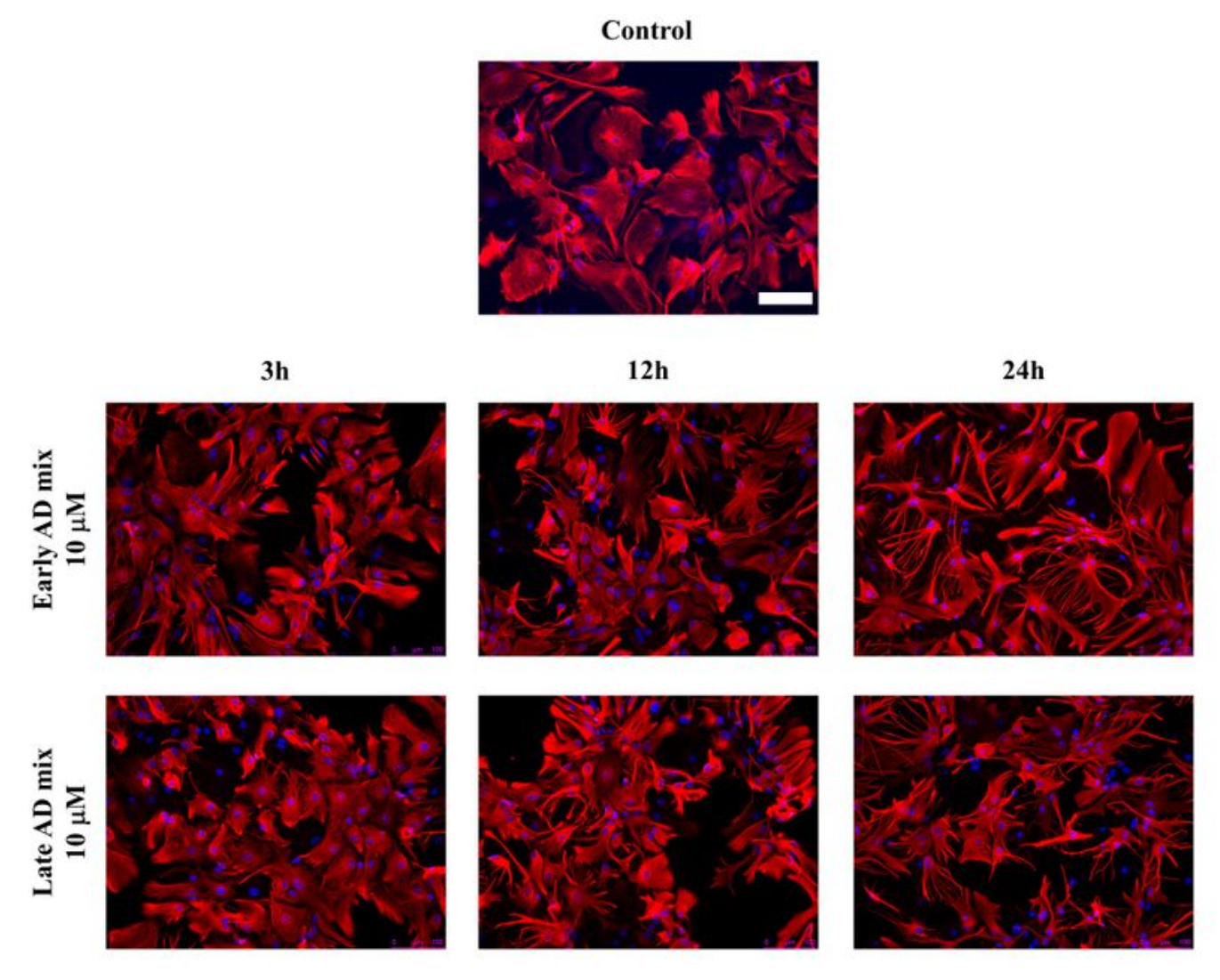

Figure 2 
Oxysterol mixtures induce a clear morphological change in astrocytes. Primary astrocytes were treated with $10 \mathrm{LM}$ of the Early or Late AD oxysterol mixture for up to $24 \mathrm{~h}$. Astrocyte morphology was examined by immunocytochemistry using a glial fibrillary acidic protein (GFAP) antibody (red) and nuclei were stained with Hoechst 33258 (blue). Representative images from three experiments are shown. Cells were imaged using an Eclipse Ti-E Microscope (Nikon; 20X objective; scale bar: 100 Dm).

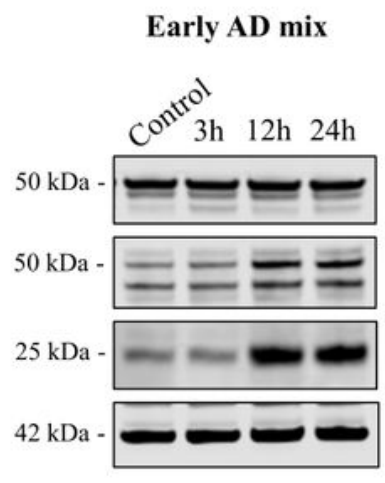

\section{Late AD mix}
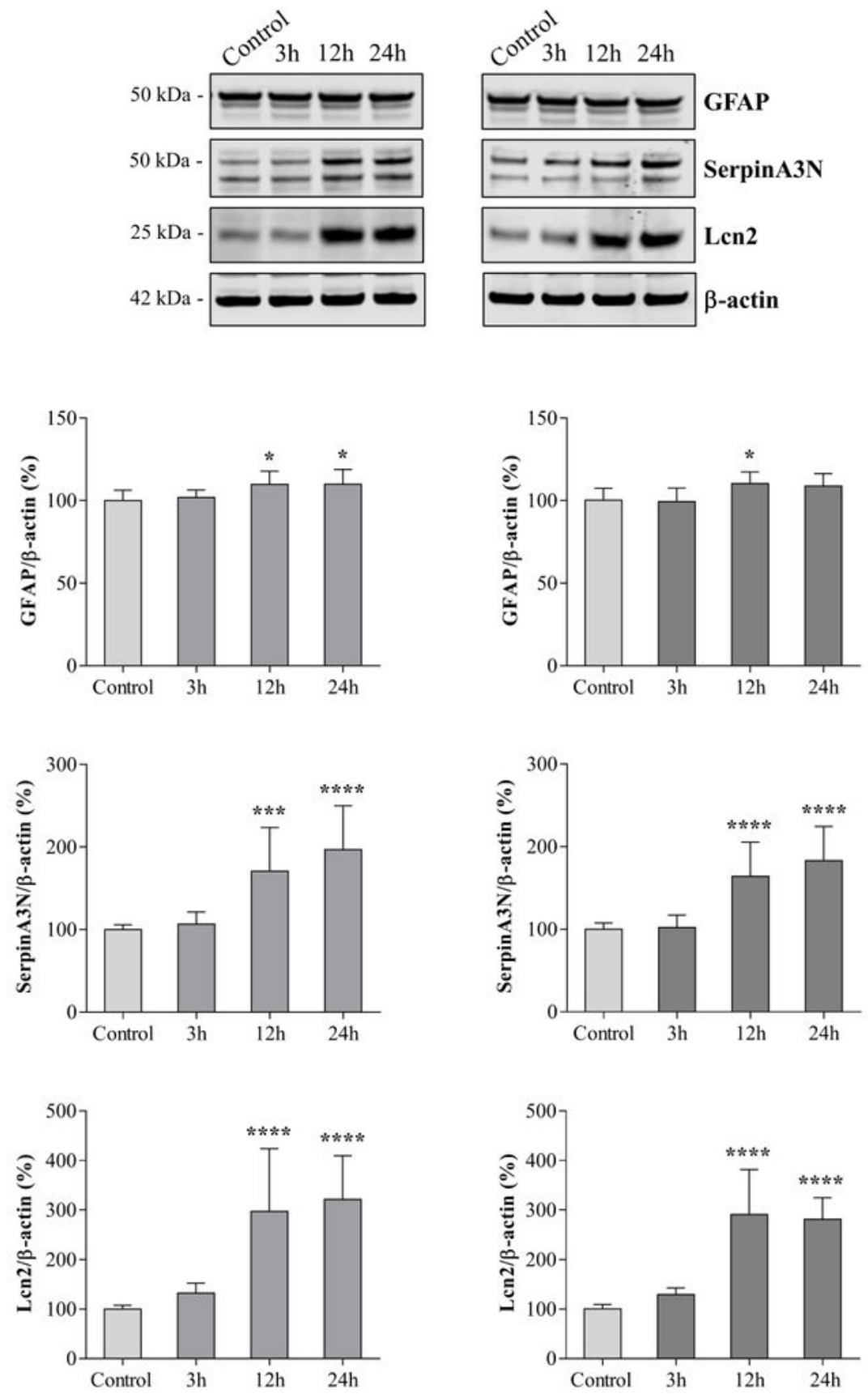

Figure 3 
Oxysterol mixtures increase the synthesis of pan-reactive astrocyte markers. The glial fibrillary acidic protein (GFAP), serine protease inhibitor A3N (SerpinA3N), and lipocalin-2 (Lcn2) protein levels were determined by Western blotting of lysates from primary astrocytes treated with $10 \mathrm{GM}$ of the Early or Late

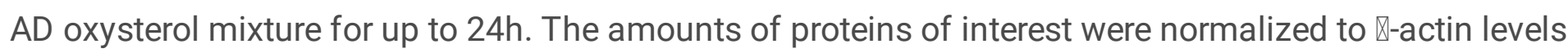
in the same sample and are represented as percentage of average control values. Data are expressed as mean values $\pm S D$ of three different experiments $\left(n=9\right.$, one-way ANOVA). ${ }^{* \star * * P<0.0001, ~}{ }^{* \star *} P<0.001$, $\star P<0.05$ vs. control.

A

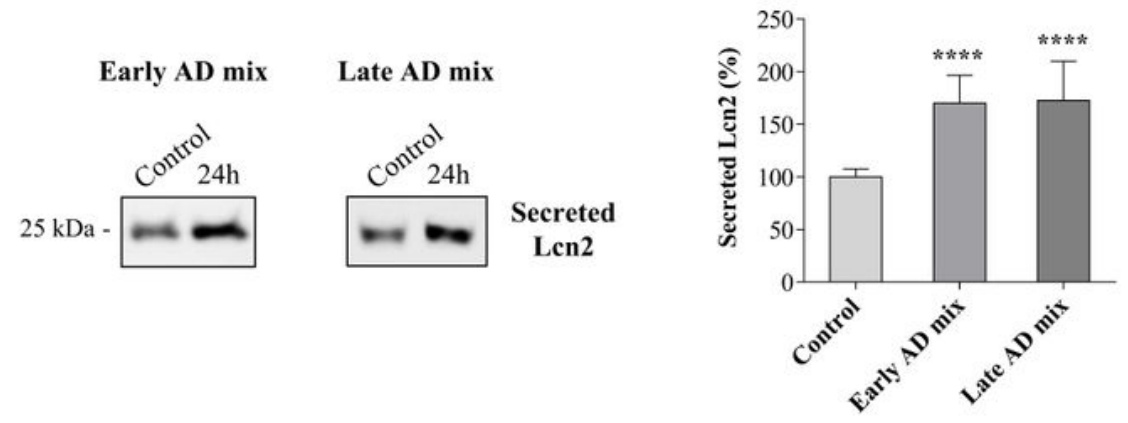

B

ACM (Late AD mix)

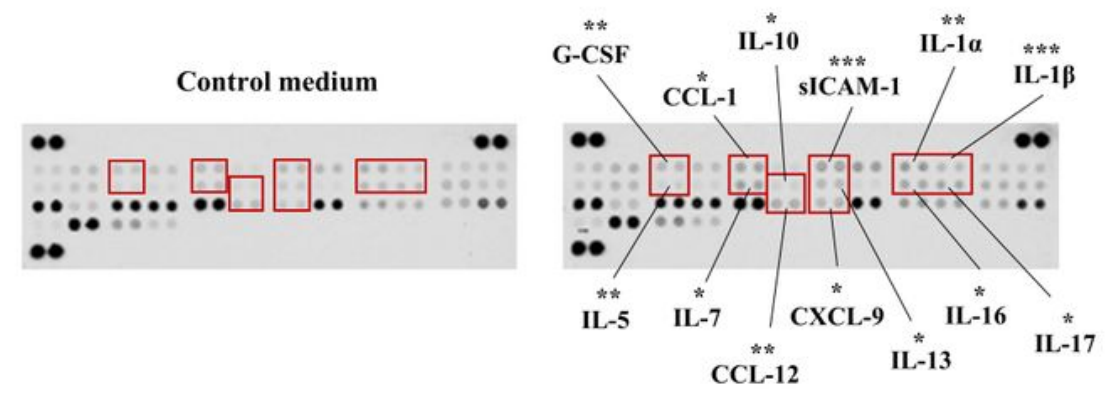

C

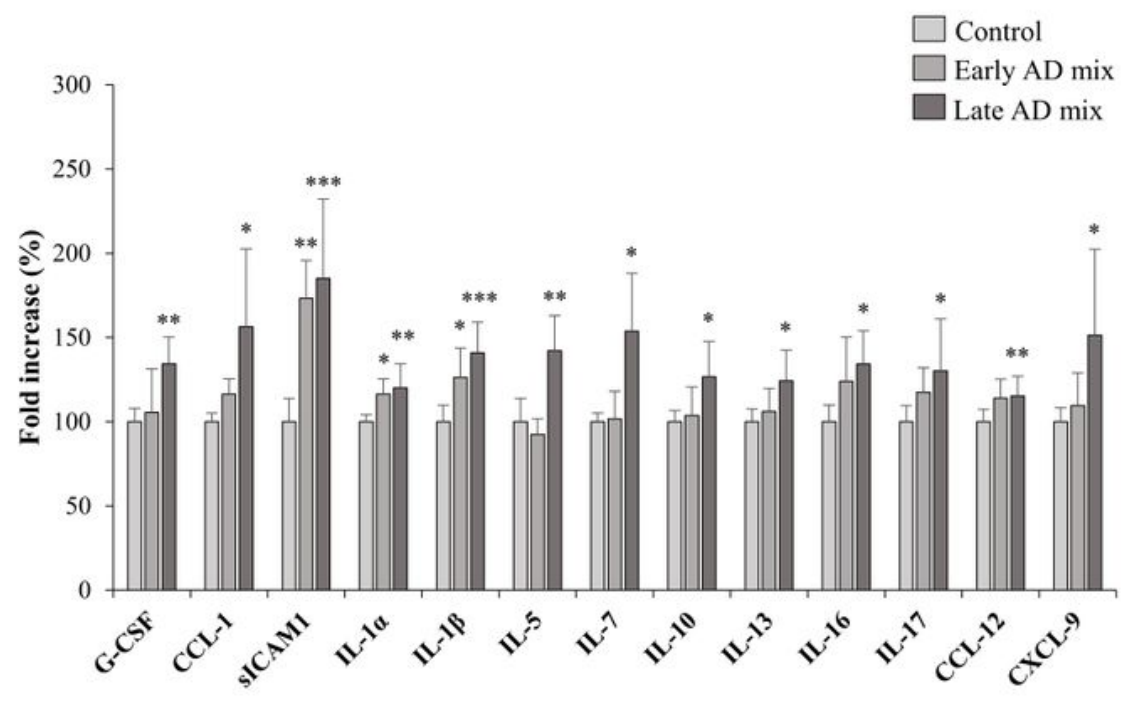




\section{Figure 4}

Oxysterol treatment increases the release of lipocalin-2 ( $\mathrm{Lcn} 2)$, cytokines and chemokines from astrocytes. Astrocyte cultures were treated with the Early or Late AD oxysterol mixture (10 DM) for 24h. (A) Lcn2 protein levels in astrocyte conditioned media (ACM) were examined by Western blotting. Data are expressed as mean values \pm SD from three different experiments and are shown as percentage change from average control values ( $n=9$, one-way ANOVA). ${ }^{* \star *} P<0.0001$ vs control. (B) Mouse cytokine profiler antibody arrays were used to detect the amounts of 40 mediators in ACM. Representative images of array membranes are shown, highlighted some of the cytokines found to be significantly increased in ACM from treated cells. The bar chart shows the cytokines significantly affected by oxysterol mixture treatments as percentage change from control. Data are expressed as mean values \pm SD from three different experiments $\left(n=6\right.$, one-way ANOVA). ${ }^{* \star *} P<0.001,{ }^{*} P<0.01$, and ${ }^{*} P<0.05$ vs control. 
A

$\square$ Fresh medium
$\square$ Medium with oxysterols or ethanol
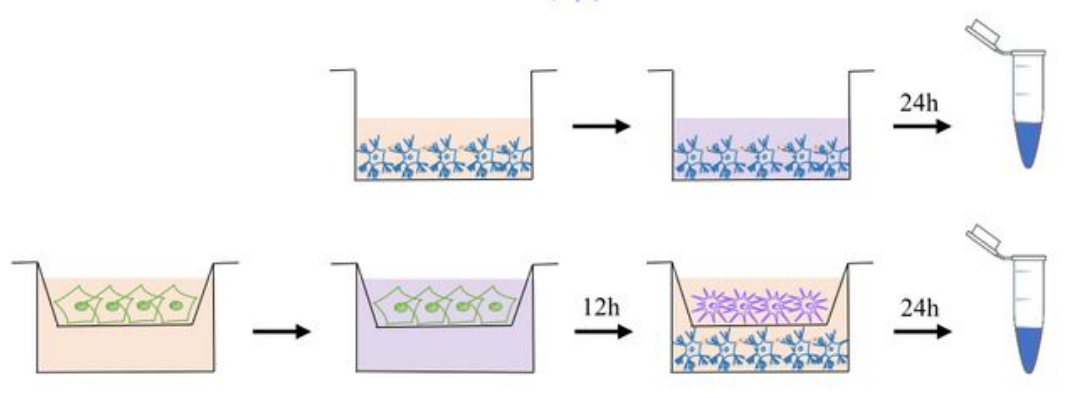

B
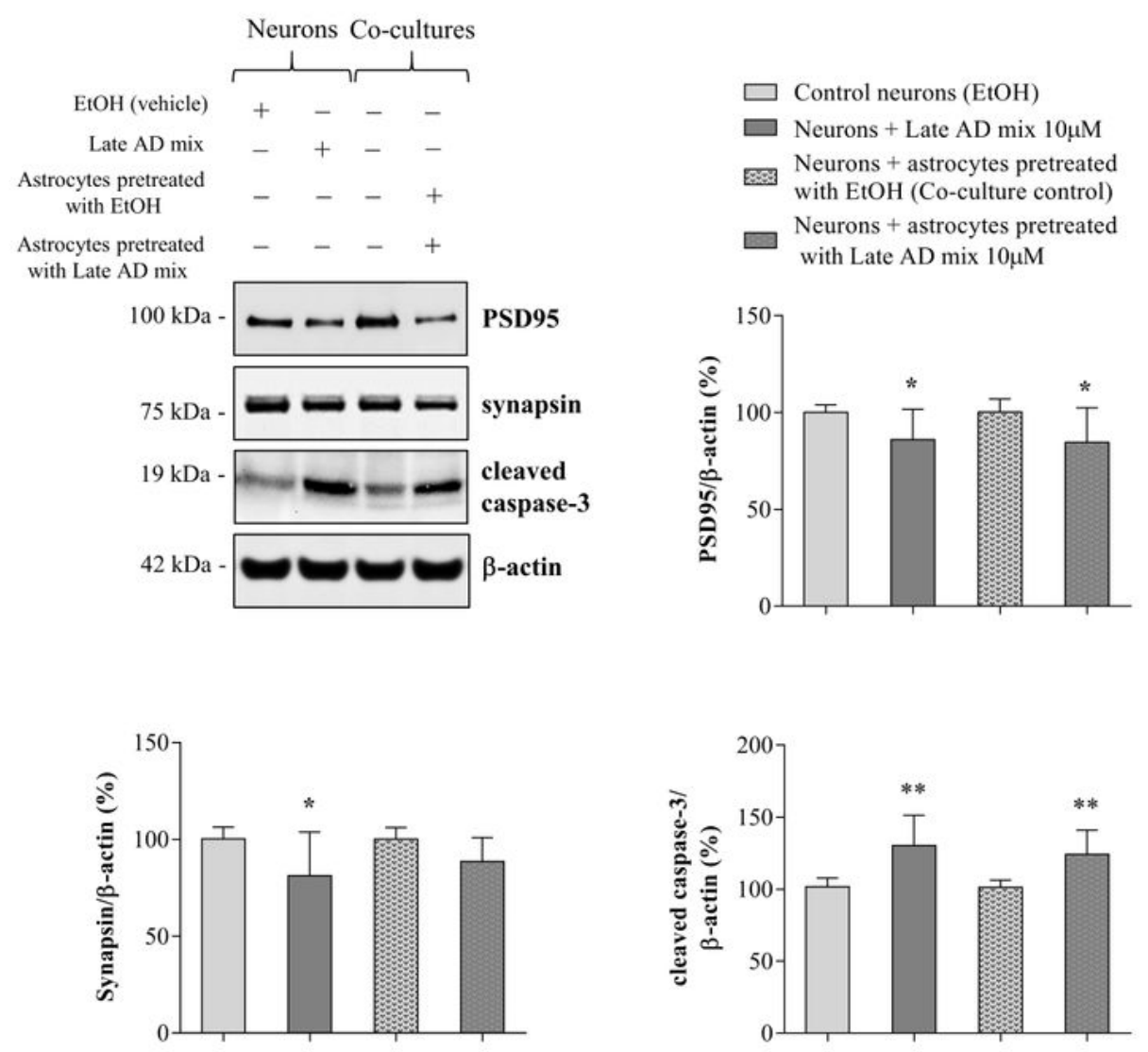

Figure 5

Oxysterol-stimulated astrocytes compromise neuronal health. Primary neurons (13 DIV) were treated with the Late $A D$ oxysterol mixture $(10 \mu \mathrm{M})$ for $24 \mathrm{~h}$ or they were co-cultured for $24 \mathrm{~h}$ with astrocytes grown on cell culture inserts that had been previously treated with the same oxysterol mixture for $12 \mathrm{~h}$. (A) Graphical representation of co-culture experiments. (B) The protein levels of the postsynaptic density protein 95 (PSD95), synapsin, and cleaved caspase-3 were determined by Western blotting. Data were normalized to 


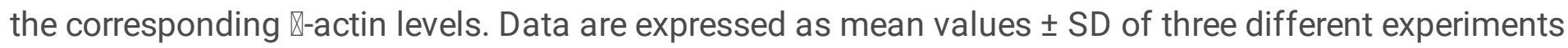
as percentage change from respective control ( $n=9$, Student's t-test). ${ }^{\star *} P<0.01$ and ${ }^{*} P<0.05$ vs control.

A

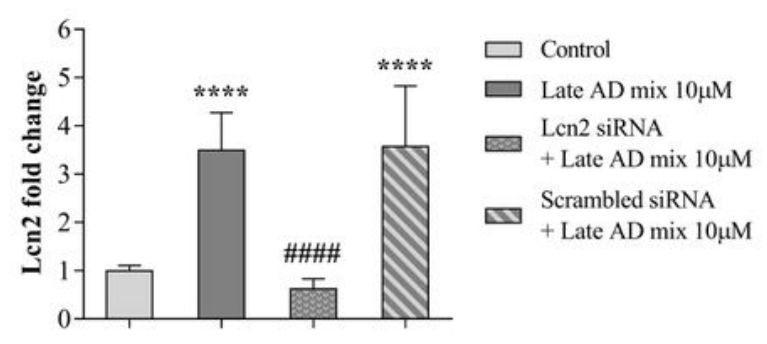

B
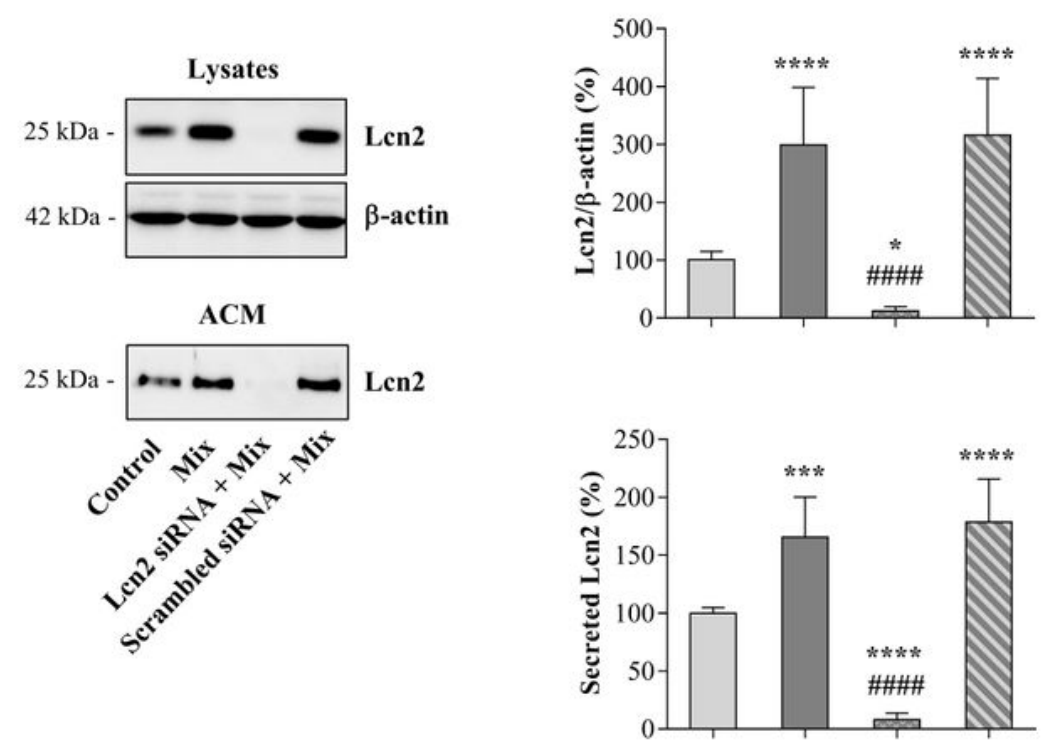

C
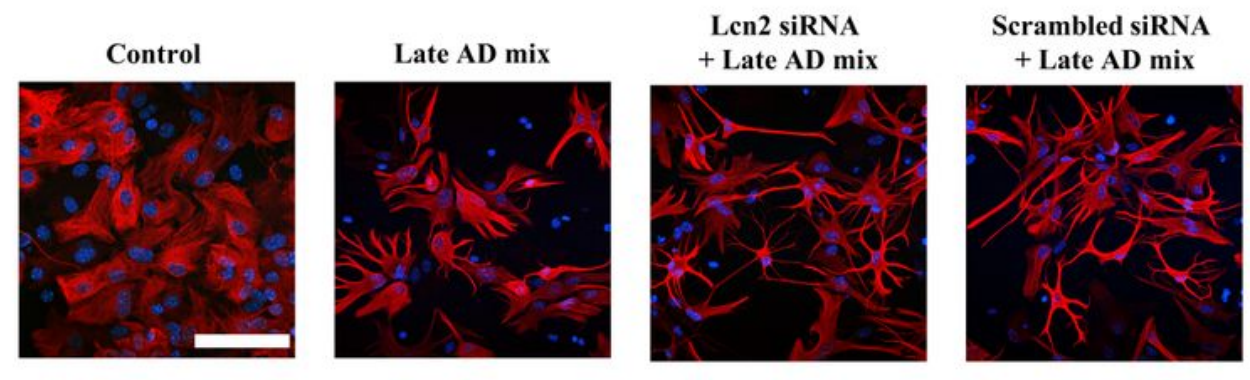

Figure 6

Lipocalin-2 ( $\operatorname{ccn} 2$ ) gene silencing prevents Lcn2 upregulation and release into the medium. Astrocyte cultures were transfected for $6 \mathrm{~h}$ with Lcn2 or scrambled siRNA and then treated with the Late AD oxysterol mixture $10 \mathrm{GM}$ (Mix) for 12h. After treatment, the medium was changed and astrocytes were 
incubated with fresh medium for 24h. Transient Lcn2 gene knockdown was evaluated by (A) real-time RTPCR and (B) Western blotting of both lysates and astrocyte conditioned media (ACM) samples. Data were

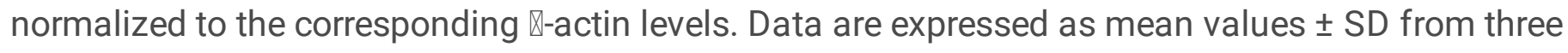
different experiments as percentage change from control ( $n=9$, one-way ANOVA). ${ }^{\star} * \star * P<0.0001$, ${ }^{* * *} P<0.001$, and ${ }^{*} P<0.05$ vs control; \#\#\#\# $P<0.0001$ vs oxysterol treated. (C) Astrocyte morphology was examined by immunocytochemistry using a glial fibrillary acidic protein (GFAP) antibody (red) and nuclei were stained with Hoechst 33258 (blue). Representative images from three experiments are shown. Cells were imaged using an LSM800 confocal microscope (Zeiss, 40X objective; scale bar: $100 \mathrm{Dm}$ ). 
A
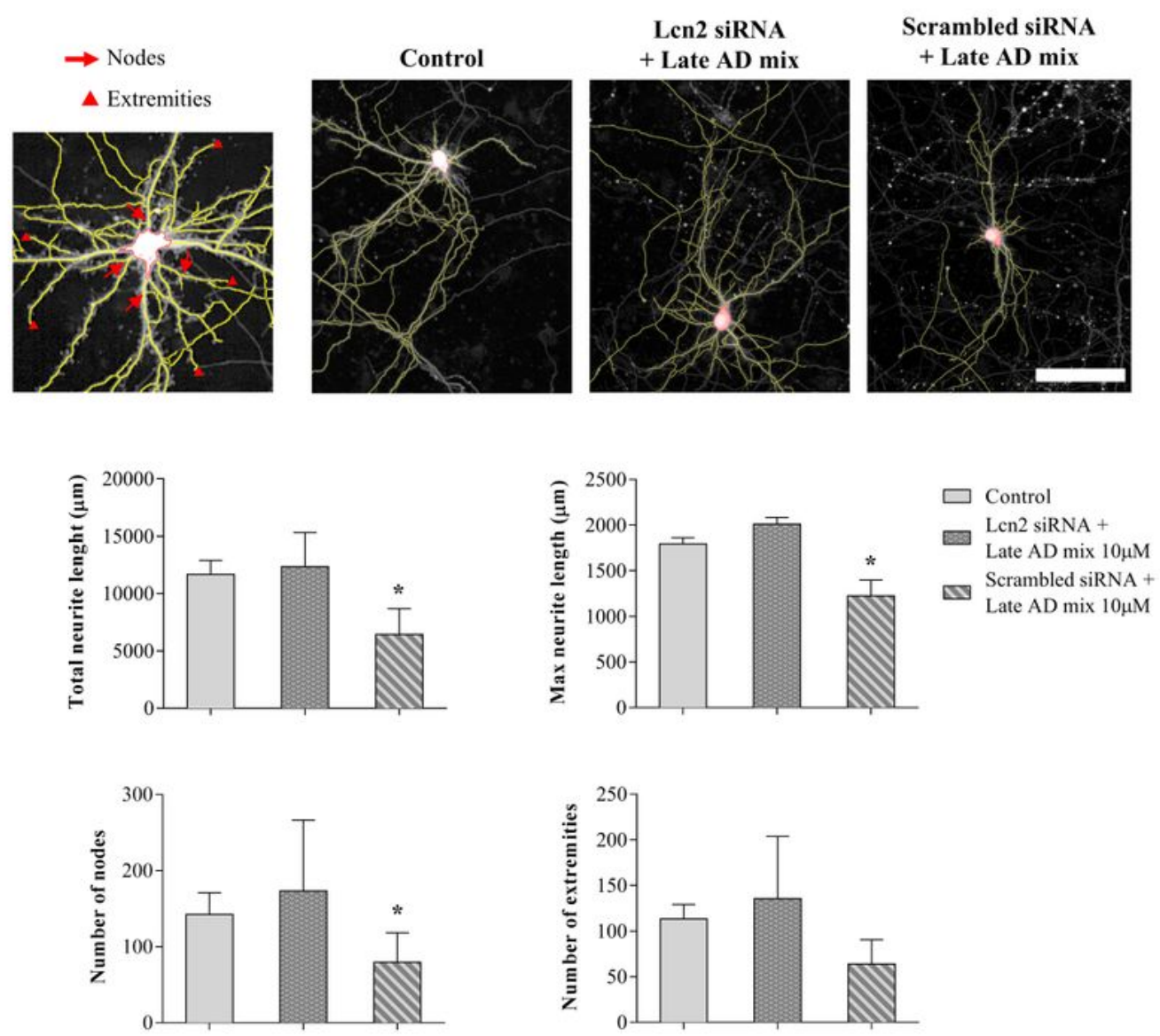

B
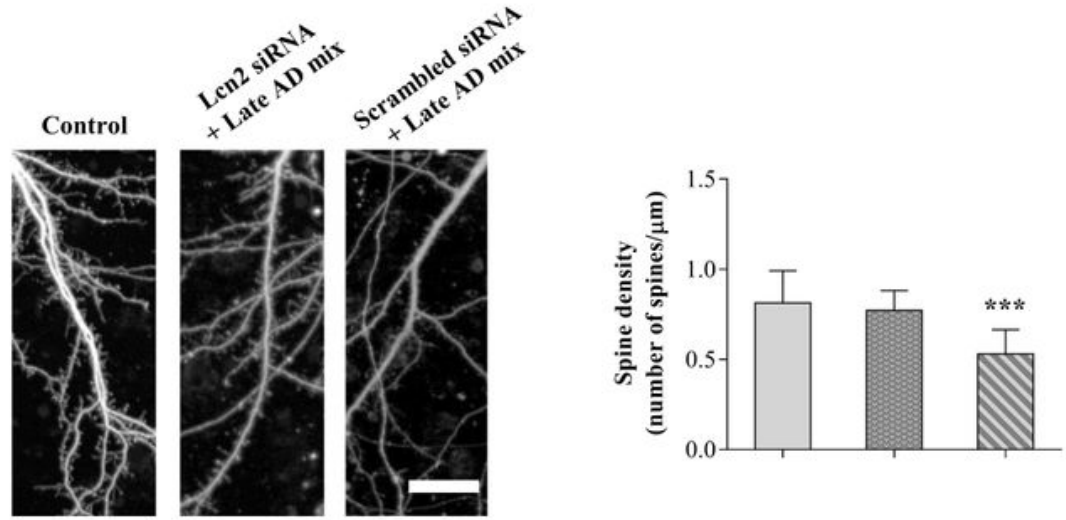

\section{Figure 7}

Lipocalin-2 (Lcn2) secreted by oxysterol-stimulated astrocytes affects neurite complexity and decreases dendritic spine density. Primary neurons were incubated for $24 \mathrm{~h}$ with conditioned media from astrocytes transfected for $6 \mathrm{~h}$ with Lcn2 or scrambled siRNA and then treated with the Late AD oxysterol mixture 10 $\square \mathrm{M}$ for $12 \mathrm{~h}$. After treatment, the medium was changed and astrocytes were incubated with fresh medium for $24 \mathrm{~h}$. High resolution digital images of live neurons were obtained using an Opera-Phenix microscope 
(Perkin-Elmer). (A) Neurite complexity was analysed using Harmony software. Total and maximum neurite length, number of nodes and extremities were quantified and compared between groups. Representative images are shown (scale bar: $100 \mathrm{Dm}$ ). Data are expressed as mean values \pm SD from three different experiments and were obtained from analysis of 20 neurons/condition (one-way ANOVA). ${ }^{*} \mathrm{P}<0.05$ vs control. (B) NeuronStudio software was used for dendritic spine analysis. Spine density was defined as number of spines per micrometer of dendrite length and was obtained from analysis of 20 neurons/condition (one-way ANOVA). Representative images are shown (scale bar: $25 \square \mathrm{m}$ ). Data are expressed as mean values \pm SD from three different experiments. ${ }^{\star * *} \mathrm{P}<0.001$ vs control.

\section{Supplementary Files}

This is a list of supplementary files associated with this preprint. Click to download.

- SupplementalFig4.tif

- SupplementalFig3.tif

- SupplementalFig2.tif

- SupplementalFig1.tif 